Pacific Journal of Mathematic 


\title{
THE HOMOTOPY GROUPS OF KNOTS I. HOW TO COMPUTE THE ALGEBRAIC 2-TYPE
}

\author{
S. J. LOMONACO, JR.
}

Dedicated to the Memory of R. H. Fox

Let $K$ be a CW complex with an aspherical splitting, i.e., with subcomplexes $K_{-}$and $K_{+}$such that (a) $K=K_{-} \cup K_{+}$ and (b) $K_{-}, K_{0}=K_{-} \cap K_{+}, K_{+}$are connected and aspherical. The main theorem of this paper gives a practical procedure for computing the homology $H_{*} \widetilde{K}$ of the universal cover $\widetilde{K}$ of $K$. It also provides a practical method for computing the algebraic 2-type of $K$, i.e., the triple consisting of the fundamental group $\pi_{1} K$, the second homotopy group $\pi_{2} K$ as a $\pi_{1} K$-module, and the first $k$-invariant $k K$.

The effectiveness of this procedure is demonstrated by letting $K$ denote the complement of a smooth 2 -knot $\left(S^{4}, k S^{2}\right)$. Then the above mentioned methods provide a way for computing the algebraic 2-type of 2-knots, thus solving problem 36 of R. H. Fox in his 1962 paper, "Some problems in knot theory." These methods can also be used to compute the algebraic 2-type of 3-manifolds from their Heegaard splittings. This approach can be applied to many other well known classes of spaces. Various examples of the computation are given.

Table of Contents

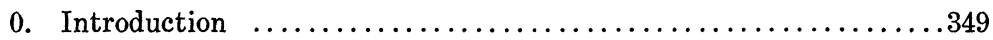

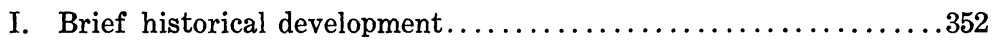

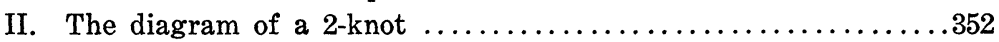

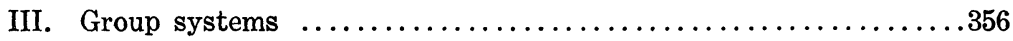

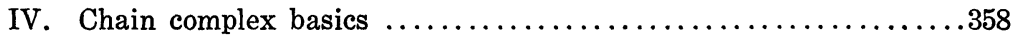

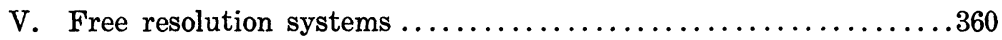

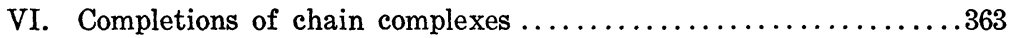

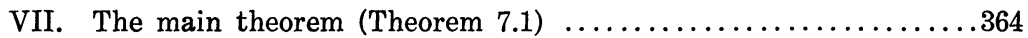

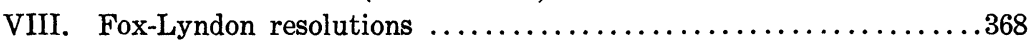

IX. A constructive form of the main theorem (Theorem 9.2)......369

$\mathrm{X}$. What is the significance of the algebraic 2-types of 2 -knots?...373

XI. Miscellaneous corollaries on 2 -knots .................... 375

XII. Conclusion: Problems and more problems $\ldots \ldots \ldots \ldots \ldots \ldots \ldots 376$

Appendix A. A computing manual for algebraic 2-type calculations....378

Appendix B. Explicit calculations for 2 -knots $\ldots \ldots \ldots \ldots \ldots \ldots \ldots 383$

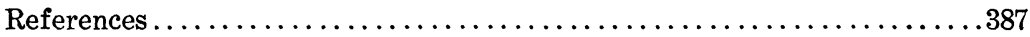

o. Introduction. Let $K$ be a $\mathrm{CW}$ complex with an aspherical splitting. An aspherical splitting of a CW complex $K$ is a triad $\left(K, K_{-}, K_{+}\right)$of $\mathrm{CW}$ complexes such that (a) $K=K_{-} \cup K_{+}$and (b) 
$K_{-}, K_{0}=K_{-} \cap K_{+}, K_{+}$are connected and aspherical. ${ }^{1}$ The main theorem (Theorem 7.1) of this paper gives an effective procedure for computing the singular homology $H_{*} \widetilde{K}$ of the universal cover $\widetilde{K}$ of $K$ from the groups $\pi_{1} K_{-}, \pi_{1} K_{0}, \pi_{1} K_{+}$and the morphisms $g_{ \pm}: \pi_{1} K_{0} \rightarrow \pi_{1} K_{ \pm}$induced by inclusion. It also provides a practical method for computing the algebraic 2-type of $K$, i.e., the triple consisting of the fundamental group $\pi_{1} K$, the second homotopy group $\pi_{2} K$ as a $\pi_{1} K$-module, and the first $k$-invariant $k K$ lying in the cohomology group $H^{3}\left(\pi_{1} K ; \pi_{2} K\right)$.

The effectiveness of this procedure is demonstrated by letting $K$ denote the complement of a smooth (or locally flat piecewise linear (PL)) 2-knot $\left(S^{4}, k S^{2}\right)$. Then the above mentioned method provides a way of computing the algebraic 2-type of 2-knots from their hyperbolic splittings (def. 2.1). This solves problem 36 of R.H. Fox in his 1962 paper [22], and substantially more.

The effectiveness of this procedure is also demonstrated by letting $K$ denote a closed connected 3-manifold. Then the algebraic 2-type of $K$ can be computed from any one of its Heegaard splittings (of positive genus). The methods of this paper apply to complements of unsplittable 2-links and to many other types of spaces. Various examples of this computational procedure are given in Appendix B.

A 2-knot is said to be quasi-aspherical (QA) if the third homology group of the universal cover of its complement $K$ vanishes (def. 10.1). It is demonstrated that the homotopy type of the complement $K$ of a QA 2-knot is completely determined by the algebraic 2-type of $K$. This suggests a possible analogue in dimension four of Papakyriakopoulos' theorem on the asphericity of 1-knots [53]. (See Problem 1 in $\S$ XII.)

It is demonstrated that, for every homology 1-sphere $K$ (hence, for all $n$-knot complements), $H_{3}\left(\pi_{1} K\right)=\pi_{2} K / \mathscr{F} \pi_{2} K=\left(Z \otimes_{\pi_{1} K} \pi_{2} K\right)$, where $\mathscr{F}$ denotes the augmentation ideal of $\pi_{1} K$. (This can be thought of as a generalization of the Kervaire condition $H_{2}\left(\pi_{1} K\right)=0$. See [36].) It follows that the first $k$-invariant of a homology 1 -sphere $K$ does not vanish if $H_{3}\left(\pi_{1} K\right) \neq 0$; hence, that the first $k$-invariant of a fibered 2 -knot is nonzero if its commutator subgroup is nontrivial and finite.

Notation. Throughout this paper (unless stated otherwise) $\left(S^{n+2}, k S^{n}\right)$ will always denote a smooth $n$-knot, (if $n=2$, it can be

${ }^{1}$ In [43] $K$ is called a "generalized Eilenberg-MacLane (GEM) complex. From [43], the homotopy type of the GEM complex $K$ is completely determined by the group system $G=\left\{\pi_{1} K_{-} \leftarrow \pi_{1} K \rightarrow \pi_{1} K_{+}\right\}$. Hence, the notation $K=K(G, 1)$. For details, see [43].

${ }^{2}$ In [48] S. MacLane and J.H.C. Whitehead call this the algebraic 3-type. 
locally flat PL). By the exterior of an $n$-knot $\left(S^{n+2}, k S^{n}\right)$ is meant $S^{n+2}$ with a small open tubular neighborhood of $k S^{n}$ removed. The complement is $S^{n+2}$ with $k S^{n}$ removed.

Note to reader. This paper has been written to be read in any one of three modes, i.e., as

Mode 1. A mathematical treatise. Read $\S \S I$ through XII. To understand only the main theorem (Theorem 7.1), read the caveat below and $\S$ III through VII.

Mode 2. A "handy man's manual" for computing algebraic 2-type. In this mode only a rudimentary understanding of [11] is needed. To compute the algebraic 2-type of:

(i) 2-knot complements, then read only $\S I I$, Theorem A 2.1, Observations 1 through 3 and the Hint (all of Appendix A), and Appendix B.

(ii) 3-manifolds, then read only Theorem A 2.1, Observations 1 through 3 and the Hint (all of Appendix A).

(iii) Arbitrary spaces with aspherical splittings, then read all of Appendices A and B.

Mode 3. A paper to browse through on a lazy Sunday afternoon. Use the table of contents and begin by browsing in any section. All terms not defined within a section are referenced back to their place of definition.

This paper started out to be a condensed and revised version of $[41,42]$. To the author's surprise, it has developed into much more. It basically contains all the material of $[41,42]$ with the exception of the section on identities. It also contains as its main theorem, Theorem 7.1, a distillate of the computational method hidden within the recesses of $[41,42]$. This clarification makes it possible, not only to compute the entire algebraic 2-type of 2-knot complements, but of a much larger class of spaces.

Caveat. The direct limit functor $\lim _{\rightarrow}$ defined in $\S \mathrm{V}$ does not commute with the homology functor.

ACKnowledgment. I would like to thank John Harper for his helpful discussions on the $k$-invariant. I would also like to thank John Milnor for pointing out that, as an immediate consequence of [23], all the hyperbolic points of a smooth (locally flat PL) 2-knot can be pushed into the same 3-dimensional hyperplane (see Theorem 
2.1). Finally, I would like to thank the referee for his helpful comments.

I. Brief historical development. The development of the work on the homotopy groups of knots had its beginnings in the early 1900's when Dehn and Wirtinger found a method for computing the fundamental group of 1-knots. Later in 1957, Papakyriakopoulos [53] determined all the higher homotopy groups of 1-knots by proving his famous theorem on the asphericity of knots. This theorem essentially says that all the higher homotopy groups of 1-knots vanish, and hence, that the homotopy type of a 1-knot complement is completely determined by its algebraic 1-type (i.e., by its fundamental group).

Mathematicians then turned to higher dimensional knots, seeking methods for computing their homotopy groups and looking for a higher dimensional analogue of Papakyriakopoulos' theorem on the asphericity of knots. In 1962 Fox [21] gave a way to compute the fundamental group of 2-knots from their motion picture representations. He then asked in problem 36 of [22] for a method for computing the second homotopy group (as a $\pi_{1}$-module) of locally flat PL 2-knots.

In this paper, a method is given for computing the second homotopy group as a $\pi_{1}$-module of smooth (or locally flat PL) 2 -knots, thus solving problem 36 of Fox. We then ask if the homotopy type, of a 2-knot complement is determined completely by its algebraic 2-type. Some evidence (Theorem 10.1) is given suggesting that this might indeed be true. A method for computing the complete algebraic 2-type of smooth (or locally flat PL) 2-knots is given.

REMARK. Please refer to the bibliography for a more complete historical development. (Also see [37] and [62].)

II. The diagram of a $2-k n o t$. If we are to compute the algebraic 2-type of a 2-knot, we will first need a convenient way of representing such knots. We will utilize Fox's motion picture representation of knots, i.e., a representation in terms of a parameterized family of 3-dimensional cross-sections [21]. We will then carry this method one step further by showing that essentially only one single 3-dimensional cross-section, the so called 0 -level, is needed.

As mentioned in $\S 0,\left(S^{4}, k S^{2}\right)$ will denote a smooth (or locally flat PL) 2-knot. Throughout this section $K$ will denote its $e x-$ terior, i.e., $S^{4}$ with a small open tubular neighborhood of $k S^{2}$ removed. 
For a given function $\tau:\left(S^{4}, \infty\right) \rightarrow\left(S^{1}, \infty\right)$ and for each point $t$ in the 1-sphere $S^{1}$, define the t-level as

$$
K_{t}=K \cap\left[\tau^{-1}(t) \cup \infty\right] \text {. }
$$

If the time function $\tau$ is suitably chosen, then the $t$-levels $K_{t}$ are the 3-dimensional cross-sections mentioned above.

There is no need to consider arbitrary time functions $\tau$. More particularly, we will show that we need only consider $\tau$ of the following type.

DEFINITION 2.1. A smooth (or PL) map $\tau:\left(S^{4}, \infty\right) \rightarrow\left(S^{1}, \infty\right)$ such that $\tau^{-1}(\infty)=\infty$ is a hyperbolic time function of an exterior $K$ of a 2-knot $\left(S^{4}, k S^{2}\right)$ provided:

(1) $\left.\tau\right|_{k s^{2}}$ has only finitely many critical points, all of which are nondegenerate and none of which is the point at $\infty$.

(2) All elliptic points occur at levels $K_{-1}$ and $K_{+1}$.

(3) All hyperbolic points occur at level $K_{0}$.

The spaces $K_{-}=\mathrm{U}_{t \geqq 0} K_{t}$ and $K_{+}=\bigcup_{t \leqq 0} K_{t}$ are called the bottom and top halves of $K$ respectively. The triad $\left(K, K_{-}, K_{+}\right)$is called a hyperbolic splitting of the knot.

It now follows from the work of Fox and Milnor [23] that:

THEOREM 2.1. Every smooth (or locally flat PL) 2-knot has a hyperbolic time function, and hence, a hyperbolic splitting.

A hyperbolic time function of the trivial 2-knot is shown in Figure 1. One of the spun trefoil is given in Figure 2.

A quick glance at Figures 1 and 2 will convince the reader that
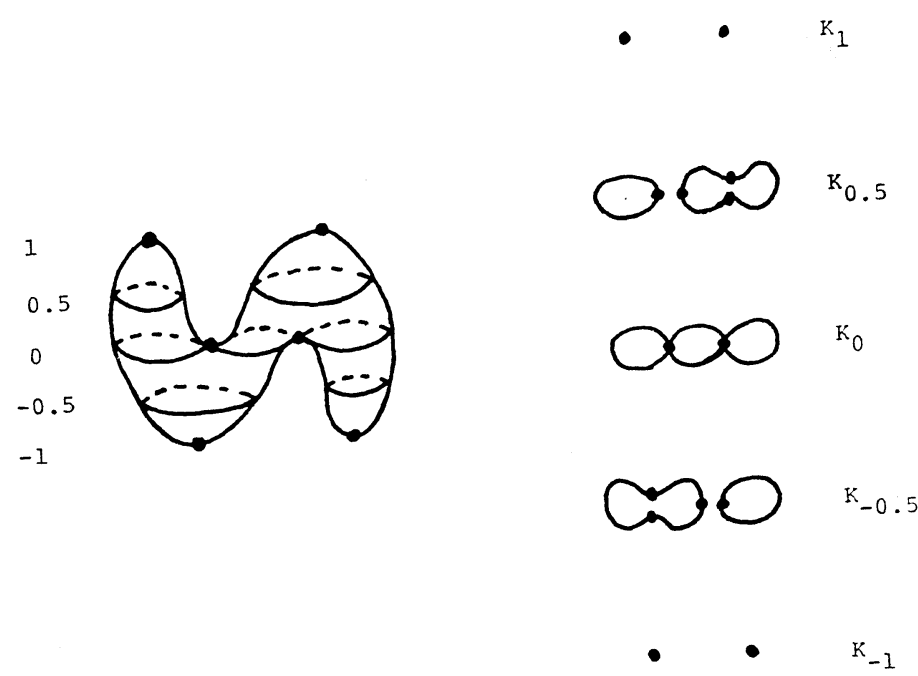

FIGURE 1. Hyperbolic time function of the trivial 2-knot. 


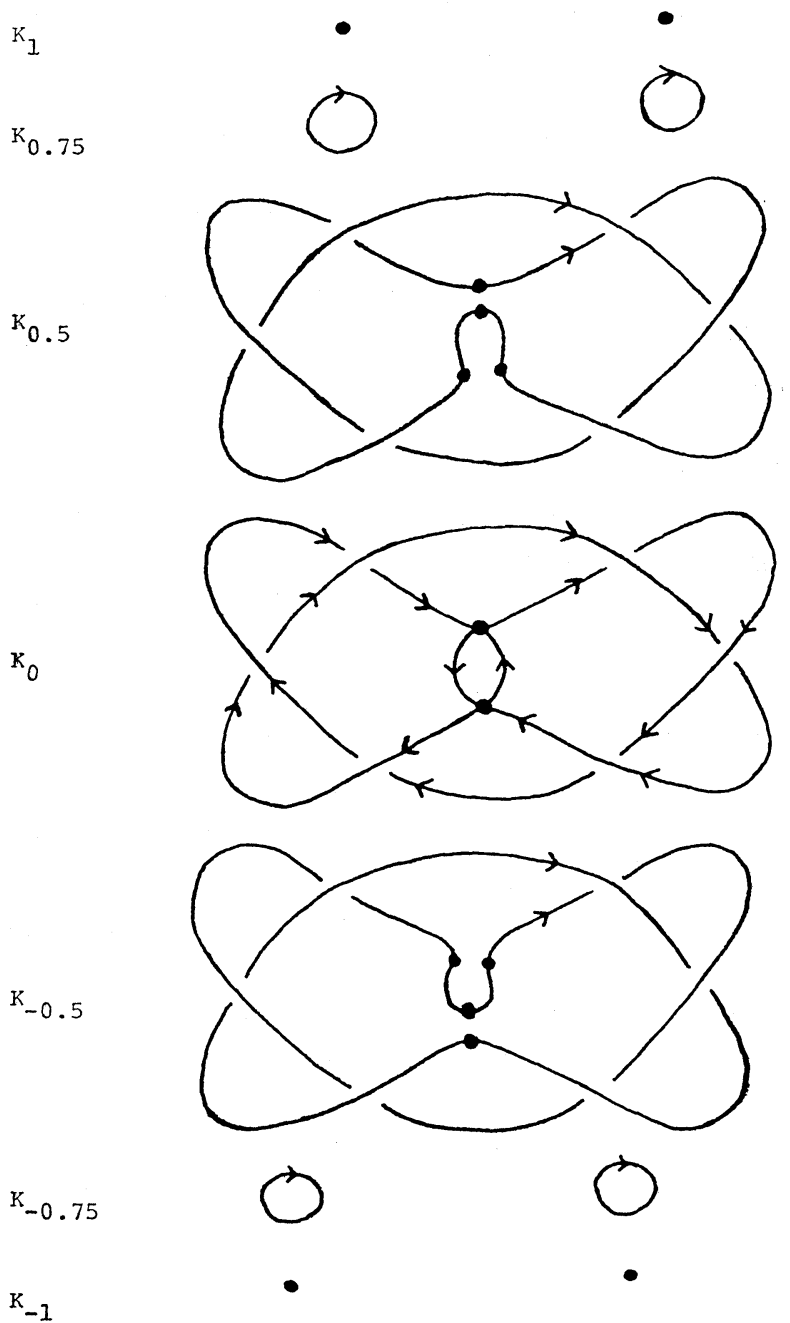

Figure 2. Hyperbolic time function of the spun trefoil.

the above method of representation is highly redundant. The entire knot can be completely reproduced from the 0 -level $K_{0}$ and a set of labels (one for each hyperbolic point) indicating how the hyperbolic points open up above, i.e., for $t>0$. We are thus led to the following convenient and compact representation of 2 -knots.

Definition 2.2. Let $K_{0}$ be a 0-level of the exterior $K$ of a smooth (or locally flat PL) 2-knot $\left(S^{4}, k S^{2}\right)$ arising from a hyperbolic time function. Then a diagram of $\left(S^{4}, k S^{2}\right)$ is a regular projection of $K_{0}$ with over and under crossings indicated in the standard way and with hyperbolic points labeled as shown in Figure 3.

By Theorem 2.1, all smooth (or locally flat PL) 2-knots have a diagram. Diagrams of the trivial 2-knot and of the spun trefoil are 


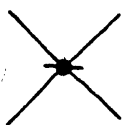

Labeled Hyperbolic Point

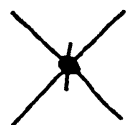

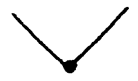

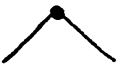

$t>0$

$t=0$

$t<0$

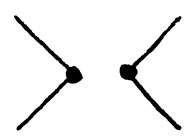

Meaning

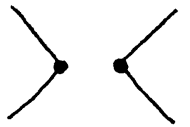

$t>0$

$t=0$

$t<0$
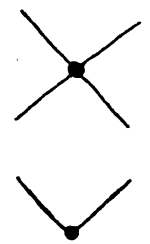

Labeled Hyperbolic Point

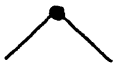

Meaning

FIGURE 3. Interpretation of hyperbolic point labels.

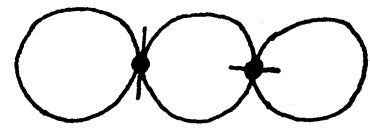

Figure 4. Diagram of trivial 2-knot.

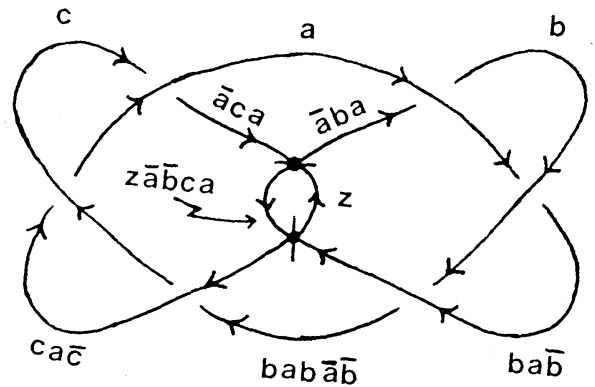

Figure 5. Diagram of Example 1, the spun trefoil.

$$
\begin{aligned}
& \pi_{1} K=\left(a, b: a b a b^{-1} a^{-1} b^{-1}=1\right) \\
& \pi_{1} K=\left(\partial U_{\square}:(1-a+b a) \partial U_{\square}=0\right) \\
& H^{3}\left(\pi_{1} K ; \pi_{2} K\right)=0 \text { and } k K=0
\end{aligned}
$$




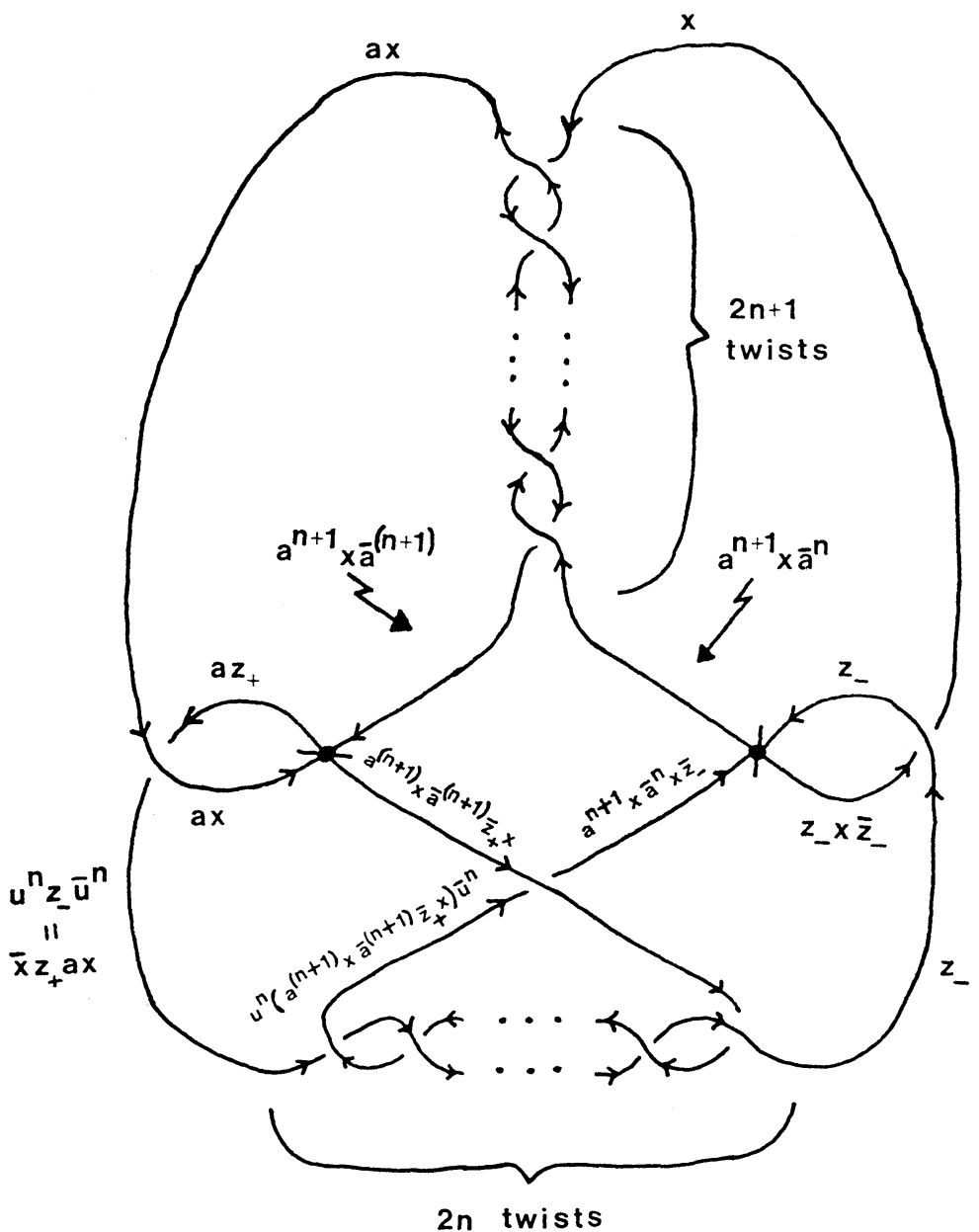

FIgURE 6. The diagram of Example 2.

$\pi_{1} K=\left(a, x: a^{3}, a x \bar{a}^{2} \bar{x}\right)$ and $\pi_{2} K=\left(\partial U_{\square}:(x-1) \partial U_{\square}=0=\left(\sum_{0}^{2 n} a^{i}\right) \partial U_{\square}\right)$ $H^{3}\left(\pi_{1} K ; \pi_{2} K\right)=Z_{2 n+1}=\left(t: t^{2 n+1}=1\right)$ and $k K=t$

shown in Figures 4 and 5 respectively. Diagrams of other 2-knots are given in Figures 6 and 7.

III. Group systems. In this section group systems and their direct limits are defined. The Van Kampen theorem is also stated. (See [10] for a more general formulation.)

A group system $G$ is a set of three groups $G_{-}, G_{0}, G_{+}$together with two group morphisms $g_{ \pm}: G_{0} \rightarrow G_{ \pm}$. If $g_{-}$and $g_{+}$are both epimorphisms (monomorphisms), then $G$ is called an epimorphic (monomorphic) group system. (The phrase "group system" is used rather than "group triad" because all definitions and results hold for a more general class of group systems.)

If $\boldsymbol{G}$ and $\boldsymbol{G}^{\prime}$ are group systems, then a morphism $\boldsymbol{f}: \boldsymbol{G} \rightarrow \boldsymbol{G}^{\prime}$ 


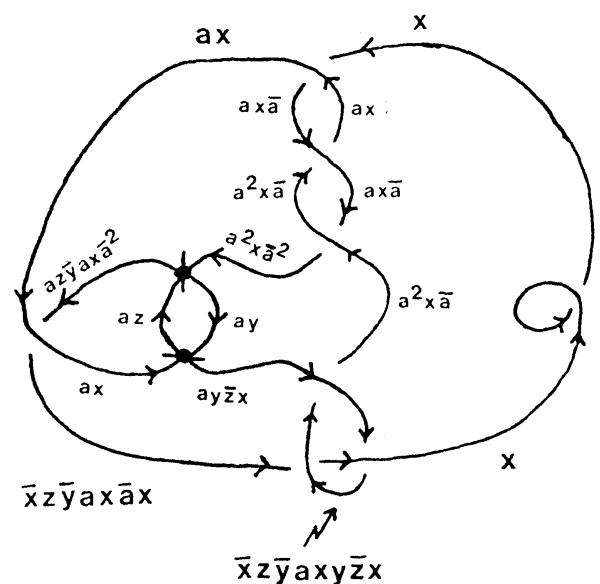

Figure 7. The diagram of Example 3.

$$
\begin{gathered}
\pi_{1} K=\left(a, x: a x=x a^{2}\right) \text { and } \pi_{2} K=\left(\partial U_{\square}:(1+a-a x) \partial U_{\square}=0\right) \\
H^{3}\left(\pi_{1} K ; \pi_{2} K\right)=0 \text { and } k K=0
\end{gathered}
$$

from $\boldsymbol{G}$ to $G^{\prime}$ is a collection of morphisms $f_{\alpha}: G_{\alpha} \rightarrow G_{\alpha}^{\prime}(\alpha=-, 0,+)$ such that the diagram

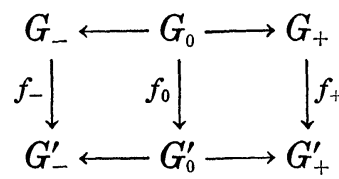

is commutative. If each morphism in $f$ is an isomorphism onto, then $\boldsymbol{f}$ is said to be an isomorphism and $G$ and $G^{\prime}$ are said to be isomorphic.

If $G$ is a group system and $G$ a group, then a morphism $f: G \rightarrow G$ from $G$ into $G$ is a collection of morphisms $f_{\alpha}: G_{\alpha} \rightarrow G$ for $\alpha=-, 0,+$ such that the diagram

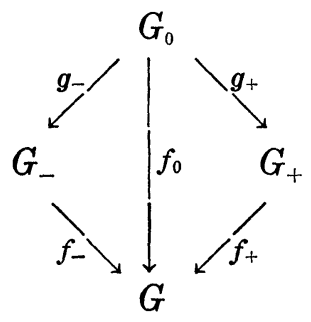

is commutative. The image of $\boldsymbol{f}$ is the subgroup of $G$ generated by the images of all the morphisms in $f$. The morphism $f$ is said to be an epimorphism if its image is $G$ itself.

Definition 3.1. A direct limit (push-out) of a group system $G$ is a group $G$ together with a morphism $f: G \rightarrow G$ such that

(1) $f$ is an epimorphism. 
(2) For every group $G^{\prime}$ and morphism $\boldsymbol{f}^{\prime}: G \rightarrow G^{\prime}$, there exists a morphism $h: G \rightarrow G^{\prime}$ such that $f^{\prime}=h f$, i.e., $f_{\alpha}^{\prime}=h f_{\alpha}$ for $\alpha=-, 0$, +. The group $G$ will be denoted by $\lim G$ and the morphism $f$ by $\boldsymbol{g}^{\infty}=\left\{g_{-}^{\infty}, g_{0}^{\infty}, g_{+}^{\infty}\right\}$.

THEOREM 3.1 [10]. Every group system has a direct limit unique to within isomorphism.

The following gives some insight into the geometric significance of the above material. More will be said in later sections.

Definition 3.2. Let $\left(Q, Q_{-}, Q_{+}\right)$be a triad of pathwise connected spaces such that $Q_{0}=Q_{-} \cap Q_{+}$is also pathwise connected. Then the group system $G$ associated with $\left(Q, Q_{-}, Q_{+}\right)$is the group system consisting of the groups $\pi_{1} Q_{-}, \pi_{1} Q_{0}, \pi_{1} Q_{+}$together with the morphisms induced by inclusion.

THEOREM 3.2. (Van Kampen) (See [10] for more general version.) Let $\left(Q, Q_{-}, Q_{+}\right)$be a triad of spaces such that

(1) $Q=Q_{-} \cup Q_{+}$.

(2) $Q_{-}, Q_{0}=Q_{-} \cap Q_{+}, Q_{+}$are open pathwise connected subspaces of the pathwise connected space $Q$.

Let $G$ be the group system associated with $\left(Q, Q_{-}, Q_{+}\right)$. Then $\pi_{1} Q=$ $\lim G$ and $g^{\infty}$ is the collection of morphisms $g_{\alpha}: \pi_{1} Q_{\alpha} \rightarrow \pi_{1} Q$ induced $\overrightarrow{b y}$ inclusion $(\alpha=-, 0,+)$.

REMARK. The main theorem of this paper (Theorem 7.1) can be thought of as a $\pi_{2}$-generalization of the above Van Kampen theorem.

IV. Chain complex basics. The mature mathematician probably will need only skim this section. The material in this section comes from $[15,47,65]$.

If $G$ is a group, then $Z G$ will denote its integral group ring; and if $g: G \rightarrow G^{\prime}$ is a group morphism, then the same symbol will denote the induced ring morphism $g: Z G \rightarrow Z G^{\prime}$. By a left (right) $G$-module is meant a left (right) $Z G$-module. For all groups $G$, the infinite cyclic group $Z$ is considered to be a left $G$-module under the trivial action. We write $\otimes_{G}$ rather than $\otimes_{Z G}$. If $M$ and $M^{\prime}$ are left $G$-modules, then a $G$-morphism $\phi: M \rightarrow M^{\prime}$ means a left $Z G$-module morphism. If $M$ and $M^{\prime}$ are left $G$-and $G^{\prime}$-modules respectively, then a map under the group morphism $g: G \rightarrow G^{\prime}$ is an additive morphism $\phi: M \rightarrow M^{\prime}$ such that $\phi(x m)=g(x) \phi(m)$ for all $x \in G$ and $m \in M$. (See [65].) 
A $G$-chain complex $C$ is a sequence of left $G$-modules $C_{q}$ for each integer $q$ together with $G$-morphisms $\partial_{q}$ : $C_{q} \rightarrow C_{q-1}$ such that (1) $C_{q}=0$ for $q<0$ and (2) $\partial_{q+1} \partial_{q}=0$ for all $q . C$ is said to be free if $C_{q}$ is a free left $G$-module for all $q$. An augmentation $\varepsilon$ of $C$ is a $G$-morphism $\varepsilon: C_{0} \rightarrow Z$ such that $\varepsilon \partial_{0}=0$ and $\varepsilon\left(x c_{0}\right)=\varepsilon\left(c_{0}\right)$ for all $x \in G$ and $c_{0} \in C_{0}$. $\mathrm{C}$ is said to be augmentable if there exists an augmentation for $C$. An augmentable $G$-chain complex $C$ together with an augmentation is called an augmented G-chain complex (which is also denoted by the same symbol $C$ ).

If $C$ and $C^{\prime}$ are augmented $G$-chain complexes, then a chain map $\phi: C \rightarrow C^{\prime}$ defined in dimensions up to $q$ is a sequence of $G$ morphisms $\phi_{i}: C_{\imath} \rightarrow C_{i}^{\prime} 0 \leqq i \leqq q$ such that

$$
\partial_{2}^{\prime} \phi_{i}=\phi_{i-1} \partial_{\imath}(1 \leqq i \leqq q) \text { and } \varepsilon^{\prime} \dot{\phi}_{0}=\varepsilon \text {. }
$$

Given two chain maps $\phi, \psi: C \rightarrow C^{\prime}$ defined up to dimension $q$, a chain homotopy $D$ from $\phi$ to $\psi$ in dimensions not greater than $q$, written

$$
D: \phi \cong \psi(\operatorname{dim} \leqq q)
$$

is a sequence of $G$-morphisms $D_{i}: C_{i} \rightarrow C_{i+1}^{\prime} 0 \leqq i \leqq q$ such that

$$
\psi_{i}=\dot{\phi}_{i}+\partial_{i+1}^{\prime} D_{i}+D_{\imath-1} \partial_{i} \quad(0 \leqq i \leqq q),
$$

where for $i=0, D_{i-1} \partial_{2}$ is omitted. If such a chain homotopy exists, then $\phi$ and $\psi$ are said to be chain homotopic in dimensions not greater than $q$, written $\dot{\phi} \cong \psi(\operatorname{dim} \leqq q)$. Two chain maps homotopic in dimensions not greater than $q$ induce the same homomorphism of the integral (reduced) homology groups for $0 \leqq i<q$.

A chain map $\phi: C \rightarrow C^{\prime}$ defined up to dimension $q$ is said to be a chain equivalence in dimensions not greater than $q$ if there exists a chain map $\phi^{\prime}: C^{\prime} \rightarrow C$ defined up to dimension $q$ such that

$$
\phi \phi^{\prime} \cong 1^{\prime}(\operatorname{dim} \leqq q) \text { and } \phi^{\prime} \phi \cong 1(\operatorname{dim} \leqq q)
$$

where 1 and $1^{\prime}$ denote the identity chain maps on $C$ and $C^{\prime}$ respectively. Such a chain equivalence induces an isomorphism of the integral (reduced) homology groups for $0 \leqq i<q$.

Definition 4.1. Let $C^{\prime}$ be a (augmented) free $G$-chain subcomplex of a (augmented) free $G$-chain complex $C$. Then $C^{\prime}$ is a proper free subcomplex of $C$ or $C$ is a proper extension of $C^{\prime}$ provided for all $q$

(1) $C_{q}^{\prime}$ is a $G$-direct summand of $C_{q}$, and

(2) $C_{q} / C_{q}^{\prime}$ is a free left $G$-module. 
(Note: The boundary operator $\partial$ of $C$ does not necessarily respect the direct sum decomposition $C_{q}=C_{q}^{\prime} \oplus\left(C_{q} / C_{q}^{\prime}\right)$.)

The following two theorems are a relativized versions of those found in [15].

Theorem 4.1. (Relative extension of chain maps.) Let $A, B, C$ be free G-chain complexes such that $B$ is a proper subcomplex of $A$. Let $\phi: A \rightarrow C$ be a chain map defined up to dimension $q$ and $\psi: B \rightarrow$ $C$ a chain map defined up to dimension $q+1$ and such that $\left.\phi_{i}\right|_{B}=$ $\psi_{i}$ for $i \leqq q$. Then $\phi$ extends to a chain map defined up to dimension $q+1$ such that $\left.\phi_{q+1}\right|_{B}=\psi_{q+1}$ if and only if the $q+1$ cocycle $\left.\eta_{q} \psi_{q} \partial_{A}\right|_{A / B}$ lying in $Z^{q+1}\left(A / B, H_{q} C\right)$ vanishes, where $\eta_{q}: Z_{q} C \rightarrow H_{q} C$ denotes the natural morphism.

THEOREM 4.2. (Relative extension of chain homotopics.) Let $A, B, C$ be free G-chain complexes such that $B$ is a proper subcomplex of $A$. Let $\phi, \phi^{\prime}: A \rightarrow C$ be chain maps defined for all $q$ and let $\psi, \psi^{\prime}: B \rightarrow C$ be chain maps defined for all $q$ such that $\left.\phi\right|_{B}=\psi$ and $\left.\phi^{\prime}\right|_{B}=\psi^{\prime}$. If $D: B \rightarrow C$ is a chain homotopy from $\psi$ to $\psi^{\prime}$ defined up to dimension $q+1$ and if $D^{*}: A \rightarrow C$ is a chain homotopy from $\phi$ to $\phi^{\prime}$ defined up to dimension $q$ and such that $\left.D^{*}\right|_{B}=D$, then $D^{*}$ extends to a chain homotopy from $\phi$ to $\phi^{\prime}$ defined up to dimension $q+1$ and such that $\left.D_{q+1}^{*}\right|_{B}=D_{q+1}$ if and only if the obstruction

$$
\left.\eta_{q+1}\left(\phi_{q+1}^{\prime}-\dot{\phi}_{q+1}-D_{q} \partial_{q+1}\right)\right|_{(A / B)_{q+1}} \text { lying in } Z^{q+1}\left(A / B, H_{q+1} C\right)
$$

vanishes, where $\eta_{q+1}: Z_{q+1} C \rightarrow H_{q+1} C$ denotes the natural morphism.

V. Free resolution systems. A more general and slicker formulation of this material is given in [43]. The definitions of $\S \S I I I$ and IV are assumed.

By a free resolution over a group $G$ is meant an augmented free $G$-chain complex $C$ such that

$$
\cdots \longrightarrow C_{q} \stackrel{\hat{o}_{q}}{\longrightarrow} C_{q-1} \longrightarrow \cdots \longrightarrow C_{0} \stackrel{\varepsilon}{\longrightarrow} Z \longrightarrow 0
$$

is exact. (The infinite cyclic group $Z$ is considered to be a left $G$-module under the trivial action.)

Definition 5.1. Let $\boldsymbol{G}$ be a group system. (See $\S$ III.) A free resolution system $C$ over $G$ is a set of free resolutions $C^{-}, C^{0}, C^{+}$ over $G_{-}, G_{0}, G_{+}$respectively together with maps $\gamma^{ \pm}: C^{0} \rightarrow C^{ \pm}$such that

(1) $Z G_{ \pm} \boldsymbol{\bigotimes}_{G_{0}} C^{0}$ is a proper subcomplex of $C^{ \pm}$. (See Definition 
(2) $\gamma_{q}^{ \pm}: C_{q}^{0} \rightarrow C_{q}^{ \pm}$is the map under the morphism $g_{ \pm}: G_{0} \rightarrow G_{ \pm}$ defined as the composition of the map of $C_{q}^{0}$ into $Z G_{ \pm} \otimes_{G_{0}} C_{q}^{0}$ given by

$$
c_{0} \longmapsto 1 \bigotimes_{\sigma_{0}} c_{0}
$$

together with the inclusion map of $Z G_{ \pm} \bigotimes_{G_{0}} C^{0}$ into $C^{ \pm}$.

Definition 5.2. Let $C$ and $C^{\prime}$ be free resolution systems over a group system $\boldsymbol{G}$. A chain map $\Phi: C \rightarrow C^{\prime}$ is a collection of chain maps $\phi^{\alpha}: C^{\alpha} \rightarrow C^{\prime \alpha}(\alpha=-, 0,+)$ such that

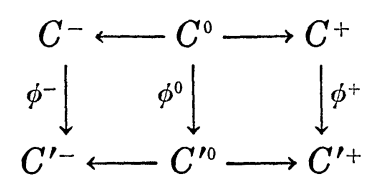

is commutative. If $\Psi: C \rightarrow C^{\prime}$ is also a chain map, then a chain homotopy $\Delta$ from $\Phi$ to $\Psi$, written $\Delta: \Phi \cong \Psi$, is a collection of chain homotopics $\Delta^{\alpha}: \phi^{\alpha} \cong \psi^{\alpha}$ such that

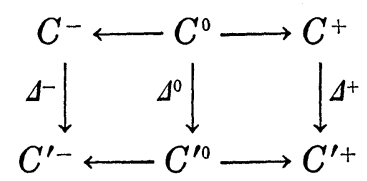

is commutative. If there is a chain equivalence from $\Phi$ to $\Psi$, we say that $\Phi$ is chain homotopic to $\Psi$, written $\Phi \cong \Psi$. A chain map $\Phi$ : $C \rightarrow C^{\prime}$ is a chain equivalence if there is a chain map $\Phi^{\prime}: C^{\prime} \rightarrow C$ such that $\Phi \Phi^{\prime} \cong 1^{\prime}$ and $\Phi^{\prime} \Phi \cong 1$, where 1 and $1^{\prime}$ denote respectively the identity chain maps on $C$ and $C^{\prime}$. $C$ and $C^{\prime}$ are said to be chain equivalent if there exists a chain equivalence from $C$ to $C^{\prime}$.

THEOREM 5.1. Any two free resolution systems over the same group system $\boldsymbol{G}$ are chain equivalent.

Proof. Let $C$ and $C^{\prime}$ be any two free resolution systems over $G$. Since $C^{0}$ and $C^{\prime 0}$ are free resolutions over $G_{0}$, there exists a chain equivalence $\phi^{0}: C^{0} \rightarrow C^{\prime 0}$ which induces a chain equivalence $\bar{\phi}^{0}$ from $Z G_{ \pm} \otimes_{G_{0}} C^{0}$ to $Z G_{ \pm} \otimes_{\alpha_{0}} C^{\prime 0}$. Since all of the obstructions of Theorems 4.1 and 4.2 vanish, $\bar{\phi}^{0}$ extends to a chain equivalence from $C^{ \pm}$to $C^{\prime \pm}$.

Definition 5.3. Let $\boldsymbol{G}$ be a group system and $\boldsymbol{C}$ be a free resolution system over $G$. Let $G=\lim G$ and let $C$ be an augmented $G$-chain complex. A chain map $\Phi: \vec{C} \rightarrow C$ is a collection of chain 
maps $\phi^{\alpha}: C^{\alpha} \rightarrow C(\alpha=-, 0,+)$ under the respective group morphisms $g_{\alpha}^{\infty}: G_{\alpha} \rightarrow G(\alpha=-, 0,+)$ such that

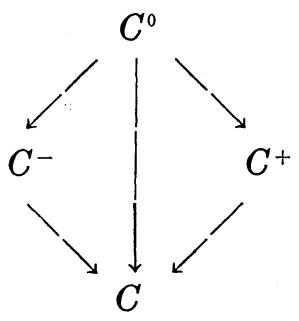

is commutative. The image of $\Phi$ is the smallest $G$-subcomplex of $C$ which contains the image of every morphism in $\Phi$. The chain map $\Phi$ is an epimorphism if its image is $C$ itself.

DEFinition 5.4. Let $C$ be a free resolution system over a group system $\boldsymbol{G}$ with $\boldsymbol{G}=\lim \boldsymbol{G}$. A direct limit (push-out) of $\boldsymbol{C}$ is

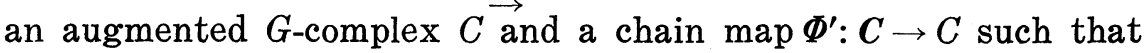

(1) $\Phi$ is an epimorphism and,

(2) For every augmented $G$-complex $C^{\prime}$ and chain map $\Phi^{\prime}: C \rightarrow$ $C^{\prime}$, there exists a chain map $p: C \rightarrow C^{\prime}$ such that $\Phi^{\prime}=p \Phi$.

Such an augmented $G$-complex $C$ will be denoted by $\lim C$ and $\Phi$ by $\Phi^{\infty}$.

The direct limit defined universally above will now be defined constructively.

Definition 5.5. Let $\boldsymbol{C}$ be a free resolution system over a group system $\boldsymbol{G}$ with $G=\lim \boldsymbol{G}$. The associated triad $\left(\widetilde{C}, \widetilde{C}^{-}, \widetilde{C}^{+}\right)$of $\boldsymbol{C}$ is

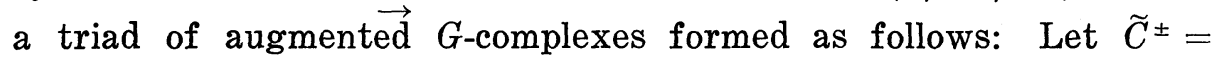
$Z G \otimes_{a_{ \pm}} C^{ \pm}$. Hence, $\widetilde{C}^{0}=Z G \otimes_{G_{0}} C^{0}=\widetilde{C}^{-} \cap \widetilde{C}^{+}$. Let $\widetilde{C}=\left(\widetilde{C}^{-} \oplus \widetilde{C}^{+}\right) / \Delta \widetilde{C}^{0}$, where $\Delta \widetilde{C}=\left\{c \oplus(-c) \mid c \in \widetilde{C}^{0}\right\} . \quad \widetilde{C}$ is called the associated complex of $C$ and $\widetilde{C}^{0}$ the associated intersection. The associated morphism $\widetilde{\Phi}: C \rightarrow \widetilde{C}$ is defined by $\tilde{\phi}_{\alpha}(c)=1_{Z G} \otimes_{G_{\alpha}} c(\alpha=-, 0,+)$, where $1_{Z G}$ denotes the identity of $Z G$.

THEOREM 5.2. Let $C$ be a free resolution system over a group system $G$. Then the direct limit of $C$ exists and is unique up to isomorphism. Moreover, the associated complex $\widetilde{C}$ together with the associated morphism, $\widetilde{\Phi}$ forms the direct limit of $\boldsymbol{C}$.

THEOREM 5.3. Let $C$ and $C^{\prime}$ be free resolution systems over the same group system $\boldsymbol{G}$. Every chain equivalence $\Phi$ from $\boldsymbol{C}$ to $\boldsymbol{C}^{\prime}$ induces a chain equivalence $\phi: \underset{\rightarrow}{\lim C}$ to $\lim _{\rightarrow} C^{\prime}$ such that 


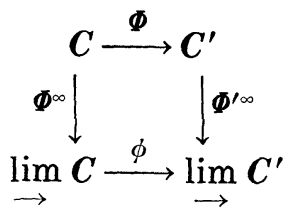

is commutative. Moreover, $\Phi$ induces a chain equivalence from the associated triad of $\boldsymbol{C}$ to the associated triad of $\boldsymbol{C}^{\prime}$. Hence, by Theorem 5.1 the direct limit of any free resolution system over $\boldsymbol{G}$ is, modulo chain equivalence, an invariant of $\boldsymbol{G}$.

The following theorem will be of use in the calculation of the algebraic 2-type of 2-knots.

THEOREM 5.4. Let $C$ be a free resolution system over a group system $G$ with $G=\lim G$. If $\phi_{\alpha}: G_{\alpha} \rightarrow G$ is an epimorphism and if $G_{\alpha}$ is of homological $\overrightarrow{d i m e n s i o n ~ a t ~ m o s t ~} q$, then

$$
Z G \bigotimes_{\sigma_{\alpha}} \operatorname{Ker} \partial_{i}^{\alpha}=\operatorname{Ker} \tilde{\partial}_{i}^{\alpha},
$$

for $i \geqq q+1$, where $\partial^{\alpha}$ and $\tilde{\partial}^{\alpha}$ denote the boundary operators of $C^{\alpha}$ in $C$ and of $\widetilde{C}^{\alpha}$ in the associated triad of $C$ respectively.

IV. Completions of chain complexes. The material given in this section will be used to define and to compute the first $k$-invariant. (See [15].)

Definition 6.1. Let $G$ be a group and $\widetilde{C}$ an augmented free $G$-chain complex. Let $q$ be a nonnegative integer such that $H_{i} \widetilde{C}=0$ for $i<q$. Then a $q$-completion $\bar{C}$ of $\widetilde{C}$ is a free resolution over $G$ such that

(1) $\bar{C}$ is a proper extension of $\widetilde{C}$ (see Def. 4.1.).

(2) $\bar{C}_{i}=\widetilde{C}_{i}$ and $\bar{\partial}_{i}=\widetilde{\partial}_{i}$ for $i \leqq q$.

Definition 6.2. Let $G, \bar{C}, \widetilde{C}$, and $q$ be as in the above definition and let id: $\bar{C} \rightarrow \widetilde{C}$ be the identity chain map defined in dimensions up to $q$. The obstruction $k(\bar{C}, \widetilde{C})$ to extending id to $\bar{C}_{q+1}$ is the $(q+1)$-cocycle

$$
k(\bar{C}, \widetilde{C})=\eta \iota \bar{\partial}: \bar{C}_{q+1} \stackrel{\bar{\partial}}{\longrightarrow} Z_{q} \bar{C} \stackrel{\iota}{=} Z_{q} \widetilde{C} \stackrel{\eta}{\longrightarrow} H_{q} \widetilde{C}
$$

lying in $Z^{q+1}\left(\bar{C}, H_{q} \widetilde{C}\right)$. (See Theorem 4.1.) Let $k(\bar{C}, \widetilde{C})$ denote the cohomology class in $H^{q+1}\left(\bar{C}, H_{q} \widetilde{C}\right)=H^{q+1}\left(G, H_{q} \widetilde{C}\right)$ represented by $k(\bar{C}, \widetilde{C})$.

The invariance of the above cohomology class is given by the following theorem. 
THEOREM 6.1. Let $G$ be a group and $C$ and $C^{\prime}$ augmented free G-chain complexes. Let $q$ be a nonnegative integer such that $H_{i} \widetilde{C}=$ $0=H_{i} \widetilde{C}^{\prime}$ for $i<q$ and let $\bar{C}$ and $\bar{C}^{\prime}$ be q-completions of $\widetilde{C}$ and $\widetilde{C}^{\prime}$ respectively. Then every chain equivalence from $\widetilde{C}$ to $\widetilde{C}^{\prime}$ extends to a chain equivalence from $(\bar{C}, \widetilde{C})$ to $\left(\bar{C}^{\prime}, \widetilde{C}^{\prime}\right)$. Each such equivalence induces an isomorphism of $H^{q+1}\left(\bar{C}^{\prime}, H_{q} \widetilde{C}^{\prime}\right)$ onto $H^{q+1}\left(\bar{C}, H_{q} \widetilde{C}\right)$ which carries $k\left(\bar{C}^{\prime}, \widetilde{C}^{\prime}\right)$ onto $k(\bar{C}, \widetilde{C})$. (See [15].)

VII. The main theorem. The main theorem (Theorem 7.1) will now be stated and proven. (The direct limit functor lim (Def. 5.4) does not commute with the homology functor. See caveat $\overrightarrow{i n} \S 0$.)

DEFINITION 7.1. An aspherical splitting of a CW complex $K$ is a triad $\left(K, K_{-}, K_{+}\right)$of $\mathrm{CW}$-complexes such that

(1) $K=K_{-} \cup K_{+}$.

(2) $K_{-}, K_{0}=K_{-} \cap K_{+}, K_{+}$are pathwise connected.

(3) $K_{-}, K_{0}, K_{+}$are aspherical. ${ }^{3}$

REMARK. It follows that there exist open subsets $\hat{K}_{-}, \hat{K}_{0}=\hat{K}_{-} \cap$ $\hat{K}_{+}, \hat{K}_{+}$of $K$ containing: $K_{-}, K_{0}, K_{+}$respectively as deformation retracts. Hence, the Van Kampen theorem (Theorem 3.2) can be applied. (See [10].)

THEOREM 7.1. ${ }^{4}$ Let $\left(K, K_{-}, K_{+}\right)$be an aspherical splitting of a $\mathrm{CW}$ complex $K$. Let $C$ be a free resolution system over the group system $G$ associated with the triad $\left(K, K_{-}, K_{+}\right)$. (See Defs. 3.2 and 5.1.) Let $\widetilde{K}_{-}, \widetilde{K}_{0}, \widetilde{K}_{+}$denote the respective lifts of $K_{-}, K_{0}, K_{+}$to the universal cover $\widetilde{K}$ of $K$. Then $\lim C$ is chain homotopic to the

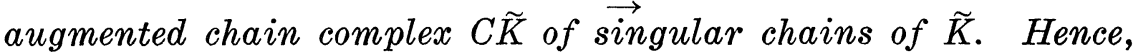

$$
\left.\bar{H}_{*} \widetilde{K} \cong H_{*} \lim C \text { (as left } Z \pi_{1} K \text {-modules }\right),
$$

where $\bar{H}$ denotes the reduced singular homology. Moreover, if $(\widetilde{C}=$ $\left.\lim C, \widetilde{C}^{-}, \widetilde{C}^{+}\right)$denotes the associated triad of $C$ (see Def. 5.5), then

$$
\bar{H}_{*} \widetilde{K}_{\alpha}=H_{*} \widetilde{C}^{\alpha}=\bar{H}_{*}\left(\pi_{1} K_{\alpha} ; Z \pi_{1} K\right) \text {, }
$$

for $\alpha=-, 0,+$. Finally, if $\bar{C}$ is a 2-completion of $\lim _{\rightarrow} C$ (See Def.

3 In [43], $K$ is called a generalized-Eilenberg MacLane (GEM) complex. The collection $\boldsymbol{K}=\left\{K_{-}, K_{0}, K_{+}\right\}$is called an aspherical structure on $K$. From [43], the homotopy type of a GEM complex is completely determined by the group system $\boldsymbol{G}=$ $\left\{\pi_{1} K_{-} \leftarrow \pi_{1} K_{0} \rightarrow \pi_{1} K_{+}\right\}$. Hence, the notation $K=K(G, 1)$. See [43] for details.

4 In [43], $\lim C$ is called the chain complex of the group system $\boldsymbol{G}=\left\{\pi_{1} K_{0} \leftarrow \pi_{1} K_{0} \rightarrow\right.$ $\left.\pi_{1} K_{+}\right\}$and denoted by $C G$; and $H_{*} \lim C=H_{*}(G ; Z G)$ is the homology of the group system $G$ with local coefficients in $Z G$, where $G=\underset{\rightarrow}{\rightarrow} G$. See [43] for details. 
6.1), then the 3-cocycle $k\left(\bar{C}, \lim _{\rightarrow} C\right)$ given by

$$
\bar{C}_{3} \stackrel{\bar{\partial}}{\longrightarrow} Z_{2} \bar{C}=Z_{2}(\stackrel{\lim }{\rightarrow} \boldsymbol{C}) \stackrel{\eta}{\longrightarrow} H_{2} \lim _{\rightarrow} \boldsymbol{C}
$$

lying in $Z^{3}\left(C, H_{2} \lim C\right)$ is a representative of the k-invariant $k K$ lying in $H^{3}\left(\bar{C}, H_{2} \underset{\lim }{\rightarrow}\right.$ ) $=H^{3}\left(\pi_{1} K ; \pi_{2} K\right)$. (See Def. 6.2)

Proof. Let $\widetilde{K}_{ \pm}, \widetilde{K}_{0}$ denotes the lifts of $K_{ \pm}, K_{0}$ respectively to the universal cover $\widetilde{K}$ of $K$ and let $\check{K}_{ \pm}$and $\check{K}_{0}$ denote respectively the universal covers of $K_{ \pm}$and $K_{0}$. Moreover, let $\sigma_{0}^{i}$ denote all the $i$-cells of $K_{0}$ and $\sigma_{ \pm}^{i}$ all the $i$-cells of $K_{ \pm}-K_{0}$. Then the $i$-cells of $\widetilde{K}, \widetilde{K}_{ \pm}, \widetilde{K}_{0}, \breve{K}_{ \pm}, \check{K}_{0}$ are respectively

$$
\begin{aligned}
& \left\{g \sigma_{-}^{i} \cup g \sigma_{0}^{i} \cup g \sigma_{+}^{i} \mid g \in \pi_{1} K\right\}, \\
& \left\{g \sigma_{0}^{i} \cup g \sigma_{ \pm}^{i} \mid g \in \pi_{1} K\right\}, \\
& \left\{g \sigma_{0}^{i} \mid g \in \pi_{1} K\right\}, \\
& \left\{g \sigma_{0}^{i} \cup g \sigma_{ \pm}^{i} \mid g \in \pi_{1} K_{ \pm}\right\}, \\
& \left\{g \sigma_{0} \mid g \in \pi_{1} K_{0}\right\} .
\end{aligned}
$$

Let $C \widetilde{K}, C \widetilde{K}_{ \pm}, C \widetilde{K}_{0}, C \check{K}_{ \pm}, C \check{K}_{0}$ denote the corresponding augmented G-chain complexes and let $\gamma^{ \pm}: C \check{K}_{0} \rightarrow C \check{K}_{ \pm}$denote the maps induced by the covering maps. Then

$$
\boldsymbol{C}=\left\{\left\{C \check{K}_{-}, C \check{K}_{0}, C \check{K}_{+}\right\},\left\{\gamma_{-}, \gamma_{+}\right\}\right\}
$$

is a free resolution system over $G$ and $\left(C \widetilde{K}, C \widetilde{K}_{-}, C \widetilde{K}_{+}\right)$is the associated triad of $\boldsymbol{C}$. Hence, by construction

$$
H_{q} \widetilde{K}=H_{q} C \widetilde{K}=H_{q} \lim _{\rightarrow} C
$$

and

$$
H_{q} \widetilde{K}_{\alpha}=H_{q} C \widetilde{K}_{\alpha}=H_{q}\left(\pi_{1} K_{\alpha} ; Z \pi_{1} K\right)
$$

for all $q$ and for $\alpha=-, 0$, + .

Next let $\bar{C}$ be a 2-completion of $\lim C$; then from $\S 6$ an obstruction $k(\bar{C}, \lim C)$ in $H^{3}\left(\bar{C}, H_{2} \lim C\right)=\vec{H}^{3}\left(\pi_{1} K ; \pi_{2} K\right)$ is defined. This construction of $k(\bar{C}, \lim C)$ is precisely the geometric definition of the $k$-invariant $k K$ given in $[14,15]$ except that the resolution $\bar{C}$ is used instead of the bar resolution.

This proves the theorem for a particular free resolution system over $\boldsymbol{G}$. Let $\boldsymbol{C}^{\prime}$ be any other free resolution system over $\boldsymbol{G}$ and let $\left(\widetilde{C}^{\prime}=\lim C^{\prime}, \widetilde{C}^{\prime-}, \widetilde{C}^{\prime+}\right)$ be the associated triad of $C^{\prime}$. Then from $\S \mathrm{V}$, the associated triad of $C^{\prime}$ is chain homotopic to $\left(C \widetilde{K}, C \widetilde{K}^{-}, C \widetilde{K}^{+}\right)$. Finally, 
let $\bar{C}^{\prime}$ be a 2-completion of $\widetilde{C}^{\prime}$. Then from $\S \mathrm{VI},\left(\bar{C}^{\prime}, \widetilde{C}^{\prime}\right)$ is chain equivalent to $(\bar{C}, C \widetilde{K})$ and the general theorem follows.

REMARK. Theorem 7.1 holds for more general aspherical splittings and group systemt. This is why the phrases "aspherical splitting" and "group system" are used above rather than "aspherical triad" and "group triad". (See [43]).

REMARK. Theorem 7.1 can be thought of as a $\pi_{2}$-generalization of Crowell's version of the Van Kampen theorem [10].

The following corollaries of Theorem 7.1 are stated more generally in [43].

CoROLlaRy 7.2. Let $\left(K, K_{-}, K_{+}\right)$be an aspherical splitting of a $\mathrm{CW}$ complex $K$ with universal cover $\widetilde{K}$. Then

$$
\begin{aligned}
\cdots \longrightarrow H_{q+1} \widetilde{K} & \longrightarrow H_{q}\left(\pi_{1} K_{0} ; Z \pi_{1} K\right) \\
& \longrightarrow H_{q}\left(\pi_{1} K_{-} ; Z \pi_{1} K\right) \oplus H_{q}\left(\pi_{1} K_{+} ; Z \pi_{1} K\right) \longrightarrow H_{q} \widetilde{K} \longrightarrow \cdots
\end{aligned}
$$

is a long exact sequence. ${ }^{5}$

From Theorem 7.1 or Corollary 7.2 we have as a corollary the following result of J.H. C. Whitehead.

Corollary 7.3. (Corollary to Theorem 5 in [66].) Let $\boldsymbol{G}$ be the group system associated with an aspherical splitting $\left(K, K_{-}, K_{+}\right)$ of a CW-complex $K$, i.e., associated with the triad $\left(K, K_{-}, K_{+}\right)$. (See Def. 3.2.) If all the morphisms in $G$ are monomorphisms, then $K$ is aspherical.

The following theorem will be of use in simplifying the calculation of the algebraic 2-type of 2-knots.

THEOREM 7.4. Let $\left(K, K_{-}, K_{+}\right)$be a hyperbolic splitting of a smooth (or locally fiat PL) 2-knot. (See Def. 2.1.) Then $K_{ \pm}$collapses to a wedge of $e_{ \pm} 1$-spheres, where $e_{-}$and $e_{+}$denote respectively the number of upper and lower elliptic points. Hence, $\pi_{1} K_{ \pm}$is free of rank $e_{ \pm}$and of homological dimension one.

5 This is a special case of the long exact sequence

$$
\cdots \longrightarrow H_{q+1}(G ; A) \longrightarrow H_{q}\left(G_{0} ; A\right) \longrightarrow H_{q}\left(G_{-} ; A\right) \oplus H_{q}\left(G_{+} ; A\right) \longrightarrow H_{q}(G ; A) \longrightarrow \cdots
$$

found in [43], where $\boldsymbol{G}$ denotes the system $\left\{G_{-} \leftarrow G_{0} \rightarrow G_{+}\right\}$with direct limit (or pushout) $G$ and $A$ is a right $Z G$-module. This is a generalization to push-outs of Swan's [43] Mayer Vietoris sequence (with local coefficients) for free products with amalgamation. See [63] for details. 
THEOREM 7.5. Let $K_{0}$ be the 0-level of a hyperbolic splitting of a smooth (or locally flat PL) 2-knot. (See Def. 2.1.) Then the fundamental group $\pi_{1} K_{0}$ of $K_{0}$ is of homological dimension at most two.

Proof. $\pi_{1} K_{0}$ is the group of an unsplittable 1-graph in $S^{3}$. Hence, by $[9,51]$ it is of homological dimension at most 2 . (See remark at end of $\S$ VIII.)

From Theorems 7.4 and 7.5 above it follows that the hyperbolic triad of a smooth (or locally flat PL) 2-knot satisfies all the hypotheses of the following theorem.

THEOREM 7.6. Let $\left(K, K_{-}, K_{+}\right)$be an aspherical splitting of a $\mathrm{CW}$ complex $K$ and let $\boldsymbol{G}$ be the associated group system. (See Def. 3.2.) Let $\widetilde{K}$ be the universal cover of $K$. If $\pi_{1} K_{-}, \pi_{1} K_{0}, \pi_{1} K_{+}$are respectively of homological dimension at most $1,2,1$, then the exact sequence of Corollary 7.2 reduces to

$$
H_{3} \widetilde{K} \cong H_{2}\left(\pi_{1} K_{0} ; Z \pi_{1} K\right)
$$

and to the short exact sequence

$$
\begin{aligned}
0 \longrightarrow H_{2} \tilde{K} \longrightarrow H_{1}\left(\pi_{1} K_{0} ; Z \pi_{1} K\right) & \longrightarrow H_{1}\left(\pi_{1} K_{-} ; Z \pi_{1} K\right) \oplus H_{1}\left(\pi_{1} K_{+} ; Z \pi_{1} K\right) \longrightarrow 0,
\end{aligned}
$$

and all the remaining reduced homology groups of $\widetilde{K}$ vanish.

The second homotopy groups of 2-knots were originally computed from the above short exact sequence $[41,42]$. The method given in this paper is a more general procedure of calculation. For 2-knots and 3-manifolds, this procedure of course reduces to the use of the above exact sequence. The more general procedure is needed to compute the $k$-invariant.

REMARK. Heegaard splittings of positive genus of connected 3-manifolds satisfy the hypotheses of Theorem 7.6.

From Theorem 7.4 and from the asphericity of 1-knots [53], we conclude:

THEOREM 7.7. Every hyperbolic splitting of a 2-knot is an aspherical splitting.

It is obvious that:

THEOREM 7.8. Every Heegaard splitting of positive genus of a closed connected 3-manifold is an aspherical splitting. 
VIII. Fox-Lyndon resolutions. We now give a method for constructing a free resolution of a group which, for our purposes, is geometrically more enlightening and computationally more economical than the bar construction. (Our construction is similar to that found in $[46,65]$.) This method of construction will be used to bridge the gap between the main theorem (Theorem 7.1) and its more constructive analogue (Theorem 9.2).

If $C$ is a (augmented) $G$-chain complex, then by a free basis of $C$, written

$$
S_{0}, S_{1}, S_{2}, \cdots,
$$

is meant a sequence $S_{q}(q=0,1,2, \cdots)$ of sets of symbols such that $S_{q}$ is a free basis of the left $G$-module $C_{q}$ for all $q \geqq 0$.

Let $\mathscr{P}=(\boldsymbol{x}: \boldsymbol{r})_{\dot{\phi}}$ be a presentation [11] of a group $G$. A FoxLyndon resolution over $G$ corresponding to $\mathscr{P}$ is a free resolution over $G$ constructed as follows:

Let $C_{0}$ be the free left $G$-module on the single symbol $P$ and let $C_{1}$ and $C_{2}$ be the free left $G$-modules on the sets $\boldsymbol{X}$ and $\boldsymbol{R}$, where $\boldsymbol{X}$ and $\boldsymbol{R}$ are sets of symbols in one-to-one correspondence with the elements of $\boldsymbol{x}$ and $\boldsymbol{r}$ respectively. Let $\varepsilon: C_{0} \rightarrow Z$ denote the extension of the morphism $\varepsilon: G \rightarrow Z$ which sends each element of $G$ to 1 and define the $G$-morphisms $\partial_{1}: C_{1} \rightarrow C_{0}, \partial_{2}: C_{2} \rightarrow C_{1}$ by

$$
\begin{array}{ll}
\partial_{1} X_{i}=\left(x_{i}-1\right) P & \forall X_{i} \in \boldsymbol{X} \\
\partial_{2} R_{j}=\sum_{i}\left(\partial r_{j} / \partial x_{i}\right) X_{i} & \forall R_{j} \in \boldsymbol{R},
\end{array}
$$

where $x_{i}-1$ and $\partial r_{j} / \partial x_{i}$ denote respectively the image in $Z G$ under $\dot{\phi}$ of $x_{i}-1$ and of the Fox derivative $[11,18] \partial r_{j} / \partial x_{i}$.

Next let $\boldsymbol{u}$ be a set of generators of the left $G$-module Ker $\partial_{2}$ and $\boldsymbol{U}$ a set of symbols in one-to-one correspondence with the elements of $\boldsymbol{u}$. Define $C_{3}$ as the free left $G$-module on the symbols $\boldsymbol{U}$ and $\partial_{3}: C_{3} \rightarrow C_{2}$ as the $G$-morphism given by

$$
\partial_{3} U_{k}=u_{k}=\sum_{j}\left(\partial U_{k} / \partial R_{j}\right) R_{j} \quad \forall U_{k} \in U,
$$

where $\partial U_{k} / \partial R_{j}$ denotes the left coefficient of $R_{j}$ in $u_{k}$.

Similarly, let $w$ be a set of generators of the left $G$-module $\operatorname{Ker} \partial_{3}$ and $W$ a set of symbols in one-to-one correspondence with the elements of $w$. Define $C_{4}$ as the free left $G$-module on the symbols $W$ and $\partial_{4}: C_{4} \rightarrow C_{3}$ as the $G$-morphism given by

$$
\partial_{4} W_{l}=w_{l}=\sum_{k}\left(\partial W_{l} / \partial U_{k}\right) U_{k} \quad\left(\forall W_{l} \in W\right),
$$

where $\partial W_{l} / \partial U_{k}$ denotes the left coefficient of $U_{k}$ in $w_{l}$. 
The free resolution $C$ over $G$ defined inductively as above is called a Fox-Lyndon resolution of $G$ corresponding to $\mathscr{P}$. The free basis (see definition above)

$$
P, X, R, U, W, \cdots
$$

is called a constructive basis of the Fox-Lyndon resolution $C$.

Finally, a q-completion $\bar{C}$ (see Def. 6.1) of an augmented free G-chain complex $\widetilde{C}$ is a Fox-Lyndon q-completion if $\bar{C}$ is a FoxLyndon resolution over $G$.

REMARK 1. The symbols $U$ correspond to identities and the $W$ to the identities among the identities. The coefficients $\partial U_{k} / \partial R_{j}$ and $\partial W_{l} / \partial U_{k}$ are actually the images of the Fox derivatives of these identities and identities of identities. Hence, the notation. (See $[41,42,65]$.)

REMARK 2. Let $Q$ be the complement of an unsplittable graph in the 3-sphere. Then $Q$ collapses to a 2 -dimensional $\mathrm{CW}$ complex $K$ containing only one 0 -cell. Let $(x: r)$ be the presentation of $\pi_{1} Q$ carried by the cells of $K$ and let $\widetilde{K}$ denote the universal cover of $K$. Then the cells of $K$ lift to $\widetilde{K}$ to form an augmentable $\pi_{1} Q$-chain complex $C \widetilde{K}$. This chain complex, when augmented, is a Fox-Lyndon resolution of $\pi_{1} Q$ corresponding to $(\boldsymbol{x}: \boldsymbol{r})$. Moreover, $C_{q} \widetilde{K}=0$ for $q>2$. Hence, $\pi_{1} Q$ is of homological dimension at most 2 .

IX. A constructive form of the main theorem. The FoxLyndon resolution defined in § VIII will now be used to give a more constructive form (Theorem 9.2) of the main theorem (Theorem 7.1).

Definition 9.1. Let $\boldsymbol{G}$ be a group system with direct limit $\boldsymbol{G}=\lim _{\rightarrow} \boldsymbol{G}$. A presentation $\left(\boldsymbol{x}_{-} ; \boldsymbol{x}_{0} ; \boldsymbol{x}_{+}: \boldsymbol{r}_{-} ; \boldsymbol{r}_{0} ; \boldsymbol{r}_{+}\right)_{\phi_{-}, \phi_{0}, \phi_{+}}$of $\boldsymbol{G}$ is a pair of morphism presentations [20] $\left(\boldsymbol{x}_{0} ; \boldsymbol{x}_{-}: \boldsymbol{r}_{0} ; \boldsymbol{r}_{-}\right)_{\phi_{0}, \phi_{-}}$and $\left(\boldsymbol{x}_{0} ; \boldsymbol{x}_{+}: \boldsymbol{r}_{0} ; \boldsymbol{r}_{+}\right)_{\phi_{0}, \phi_{+}}$ of $g_{-}: G_{0} \rightarrow G_{-}$and $g_{+}: G_{0} \rightarrow G_{+}$respectively. The subscripts $\phi_{0}$ and $\phi_{ \pm}$are usually omitted unless extra precision is needed. It follows from [10] that $\left(x_{-}, x_{0}, x_{+}: r_{-}, r_{0}, r_{+}\right)$is a presentation of $G$.

REMARK. If $f: G \rightarrow H$ is a group morphism, then a presentation $(\boldsymbol{x} ; \boldsymbol{y}: \boldsymbol{r} ; \boldsymbol{s})$ of $f$ is a set of two presentations $(\boldsymbol{x}: \boldsymbol{r})_{\phi}$ and $(\boldsymbol{x} ; \boldsymbol{y}: \boldsymbol{r} ; \boldsymbol{s})_{\psi}$ of $G$ and $H$ respectively such that

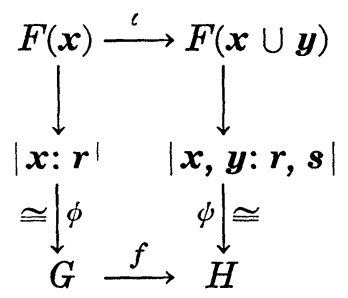


is commutative, i.e., such that $f$ is the morphism induced by the inclusion 6. (See [20].)

By arguments similar to those found in [20, p. 411], it can be shown that the following transformations do not alter the isomorphism type of the presented group system.

Definition. Let $\mathscr{P}=\left(\boldsymbol{x}_{-} ; \boldsymbol{x}_{0} ; \boldsymbol{x}_{+}: \boldsymbol{r}_{-} ; \boldsymbol{r}_{0} ; \boldsymbol{r}_{+}\right)$be a presentation of a group system $G$. Then the following are called Tietze transformations of $\mathscr{P}$.

(I) Adjoin to $r_{0}$ any one of its consequences.

$\left(\mathrm{I}_{ \pm}\right) \quad$ Adjoin to $\boldsymbol{r}_{ \pm}$any one of the consequences of $\boldsymbol{r}_{0} \cup \boldsymbol{r}_{ \pm}$.

(II) Adjoin a new symbol $x_{0}$ to $\boldsymbol{x}_{0}$ and $x_{0} u^{-1}$ to $\boldsymbol{r}_{0}$, where $u$ is in the free group $F\left(\boldsymbol{x}_{0}\right)$.

(II $\mathrm{II}_{ \pm}$Adjoin a new symbol $x_{ \pm}$to $\boldsymbol{x}_{ \pm}$and $x_{ \pm} u^{-1}$ to $\boldsymbol{r}_{ \pm}$, where $u$ is in the free group $F\left(\boldsymbol{x}_{0}, \boldsymbol{x}_{ \pm}\right)$.

By a proof similar to that given in $[19$, p. 198], it can be shown that:

THEOREM (Tietze). If $\mathscr{P}$ and $\mathscr{P}^{\prime}$ are finite presentations of the same group system, then it is possible to pass from one to the other presentation by applying a finite sequence of Tietze Transformations of types

$$
(\mathrm{I})^{ \pm 1},\left(\mathrm{I}_{-}\right)^{ \pm 1},\left(\mathrm{I}_{+}\right)^{ \pm 1},(\mathrm{II})^{ \pm 1},\left(\mathrm{II}_{-}\right)^{ \pm 1},\left(\mathrm{II}_{+}\right)^{ \pm 1} .
$$

Definition 9.2. Let $\mathscr{P}=\left(\boldsymbol{x}_{-} ; \boldsymbol{x}_{0} ; \boldsymbol{x}_{+}: \boldsymbol{r}_{-} ; \boldsymbol{r}_{0} ; \boldsymbol{r}_{+}\right)$be a presentation of a group system $G$ with $G=\lim G$. Then a Fox-Lyndon resolution system over $\boldsymbol{G}$ is a free resolution system $\boldsymbol{C}$ constructed as follows: Let $C^{0}$ be a Fox-Lyndon resolution (see $\S$ VIII) of $G_{0}$ corresponding to $\left(\boldsymbol{x}_{0}: \boldsymbol{r}_{0}\right)$. Let $C^{ \pm}$be a Fox-Lyndon resolution of $G_{ \pm}$corresponding to $\left(\boldsymbol{x}_{0}, \boldsymbol{x}_{ \pm}: \boldsymbol{r}_{0}, \boldsymbol{r}_{ \pm}\right)$formed by extending $Z G_{ \pm} \boldsymbol{\otimes}_{\sigma_{0}} C^{0}$. Then $C^{-}, C^{0}, C^{+}$together with the obvious maps $\gamma_{-}, \gamma_{+}$under the respective group morphisms $g_{-}, g_{+}$is a free resolution system over $G$. Let $P$, $\boldsymbol{X}_{0}, \boldsymbol{R}_{0}, \boldsymbol{U}_{0}, W_{0}, \cdots$ be the constructive basis (see $\S$ VIII) of $C^{0}$ and let the induced free basis (see $\S$ VIII) of $Z G_{ \pm} \otimes_{G_{0}} G^{0}$ also be denoted by the same symbols. Extend this free basis to a constructive basis $P, \boldsymbol{X}_{0} \cup \boldsymbol{X}_{ \pm}, \boldsymbol{R}_{0} \cup \boldsymbol{R}_{ \pm}, \boldsymbol{U}_{0} \cup \boldsymbol{U}_{ \pm}, \boldsymbol{W}_{0} \cup \boldsymbol{W}_{ \pm}, \cdots$ of $C^{ \pm}$. Then

$$
P ; \boldsymbol{X}_{-}, \boldsymbol{X}_{0}, \boldsymbol{X}_{+} ; \boldsymbol{R}_{-}, \boldsymbol{R}_{0}, \boldsymbol{R}_{+} ; \boldsymbol{U}_{-}, \boldsymbol{U}_{0}, \boldsymbol{U}_{+} ; \boldsymbol{W}_{-}, \boldsymbol{W}_{0}, \boldsymbol{W}_{+} ; \cdots
$$

is called a constructive basis of the Fox-Lyndon resolution system $\boldsymbol{C}$.

THEOREM 9.1. Let $\boldsymbol{C}$ be a Fox-Lyndon resolution system over a group system $\boldsymbol{G}$ corresponding to a presentation $\mathscr{P}$ of $\boldsymbol{G}$. Let $G=$ 
$\lim _{\rightarrow} G$ and $\left(\widetilde{C}, \widetilde{C}^{-}, \widetilde{C}^{+}\right)$be the associated triad (see Def. 5.5) of $C$. If

$$
P ; \boldsymbol{X}_{-}, \boldsymbol{X}_{0}, \boldsymbol{X}_{+} ; \boldsymbol{R}_{-}, \boldsymbol{R}_{0}, \boldsymbol{R}_{+} ; \boldsymbol{U}_{-}, U_{0}, U_{+} ; W_{-}, W_{0}, W_{+} ; \cdots
$$

is a constructive basis of $\boldsymbol{C}$, then

$$
\begin{aligned}
& P, \boldsymbol{X}_{0}, \boldsymbol{R}_{0}, \boldsymbol{U}_{0}, \boldsymbol{W}_{0}, \cdots \\
& P, \boldsymbol{X}_{0} \cup \boldsymbol{X}_{ \pm}, \boldsymbol{R}_{0} \cup \boldsymbol{R}_{ \pm}, \boldsymbol{U}_{0} \cup \boldsymbol{U}_{ \pm}, \boldsymbol{W}_{0} \cup \boldsymbol{W}_{ \pm}, \cdots \\
& P, \boldsymbol{X}_{-} \cup \boldsymbol{X}_{0} \cup \boldsymbol{X}_{+}, \boldsymbol{R}_{-} \cup \boldsymbol{R}_{0} \cup \boldsymbol{R}_{+}, \boldsymbol{U}_{-} \cup \boldsymbol{U}_{0} \cup \boldsymbol{U}_{+}, \boldsymbol{W}_{-} \cup \boldsymbol{W}_{0} \cup \boldsymbol{W}_{+}, \cdots
\end{aligned}
$$

are respectively the induced constructive bases of $\widetilde{C}_{0}, \widetilde{C}^{ \pm}, \widetilde{C}$, and the boundary operators are given by

$$
\begin{aligned}
& \tilde{\partial}^{0}=1_{G} \underset{G_{0}}{\underset{\partial}{\partial^{0}} \quad} \quad \tilde{\partial}^{ \pm}=1_{G} \underset{G_{ \pm}}{\boldsymbol{\partial}^{ \pm}} \partial^{ \pm} \\
& \tilde{\partial} \sigma=\left\{\begin{array}{lll}
\tilde{\partial}^{+} \sigma & \text { if } & \sigma \in \widetilde{C}^{+} \\
\tilde{\partial}^{-} \sigma & \text { if } & \sigma \in \widetilde{C}^{-},
\end{array}\right.
\end{aligned}
$$

where $\partial_{\alpha}$ is the boundary operator of $C_{\alpha}$.

THEOREM 9.2. (Constructive form of main theorem.) Let $C$ be a Fox-Lyndon resolution system over a group system $\boldsymbol{G}$ corresponding to a presentation

$$
\mathscr{P}=\left(\boldsymbol{x}_{-} ; \boldsymbol{x}_{0} ; \boldsymbol{x}_{+}: \boldsymbol{r}_{-} ; \boldsymbol{r}_{0} ; \boldsymbol{r}_{+}\right)
$$

of $\boldsymbol{G}$. Let $G=\lim \boldsymbol{G}$, let $\left(\widetilde{C}, \widetilde{C}^{-}, \widetilde{C}^{+}\right)$be the associated triad (see Def. 5.5) of $C$, and let $\bar{C}$ be a Fox-Lyndon 2-completion of $\widetilde{C}$ (see $\S$ VIII). Let

$$
P ; X_{-}, X_{0}, X_{+} ; R_{-}, R_{0}, R_{+} ; U_{-}, U_{0}, U_{+} ; W_{-}, W_{0}, W_{+} ; \cdots
$$

be a constructive basis of $\boldsymbol{C}$ and let

$$
\begin{gathered}
P, \boldsymbol{X}_{-} \cup \boldsymbol{X}_{0} \cup \boldsymbol{X}_{+}, \boldsymbol{R}_{-} \cup \boldsymbol{R}_{0} \cup \boldsymbol{R}_{+}, \boldsymbol{U}_{-} \cup \boldsymbol{U}_{0} \cup \boldsymbol{U}_{\square} \cup \boldsymbol{U}_{+}, \\
\boldsymbol{W}_{-} \cup \boldsymbol{W}_{0} \cup \boldsymbol{W}_{\square} \cup \boldsymbol{W}_{+}, \cdots
\end{gathered}
$$

be a constructive basis of $\bar{C}$ formed by extending the free basis of $\widetilde{C}$ mentioned in Theorem 9.1. Then $\mathrm{H}_{2} \widetilde{C}$ as a left G-module is generated by the elements of $\partial \boldsymbol{U}_{\square}$ and

$$
\left(\partial U_{\square}: \sum_{U_{\square} \in U_{\square}}\left(\partial W_{\square} / \partial U_{\square}\right) \partial U_{\square}=0, \forall W_{\square} \in W_{\square}\right)
$$

is a presentation of $\mathrm{H}_{2} \widetilde{C}$ as a left $Z G$-module. Moreover, if $\boldsymbol{W}^{\prime}$ is any set of generators of the left $Z G$-module $\bar{C}_{4}$, then

$$
\left(\partial \boldsymbol{U}_{\square}: \sum_{l^{\prime} \square_{\square} \in \boldsymbol{U}_{\square}}\left(\partial W^{\prime} / \partial U_{\square}\right) \partial U_{\square}=0, \forall W^{\prime} \in W^{\prime}\right)
$$

is also a presentation of $\mathrm{H}_{2} \widetilde{C}$ as a left ZG-module. Moreover, the 
obstruction $k(\bar{C}, \widetilde{C})$ lying in $H^{3}\left(\bar{C}, H_{2} \widetilde{C}\right)$ is represented by the equivariant 3-cocycle $k: \bar{C}_{3} \rightarrow H_{2} \widetilde{C}$ given by

$$
k\left(U^{*}\right)= \begin{cases}\partial U^{*} & \text { if } U^{*} \in \boldsymbol{U}_{\square} \\ 0 & \text { if } U^{*} \in \boldsymbol{U}_{-} \cup \boldsymbol{U}_{0} \cup \boldsymbol{U}_{+} .\end{cases}
$$

Proof. From the long exact sequence induced by the short exact sequence

$$
0 \longrightarrow \widetilde{C} \longrightarrow \bar{C} \longrightarrow \bar{C} / \widetilde{C} \longrightarrow 0
$$

we have that

$$
H_{2} \widetilde{C} \cong H_{3}(\bar{C} / \widetilde{C})
$$

as left $G$-modules under a isomorphism denoted by $\partial_{*} \cdot{ }^{6}$ But

$$
\phi, \phi, \phi, U_{\square}, W_{\square}, \cdots
$$

is a constructive basis of $\bar{C} / \widetilde{C}$. Hence, $U_{\square}$ is a set of generators of the left $Z G$-module $Z_{3}(\bar{C} / \widetilde{C})$ of relative 3-cycles and

$$
\partial W_{\square}=\sum_{U_{\square} \in U_{\square}}\left(\partial W_{\square} / \partial U_{\square}\right) U_{\square}
$$

is a set of generators of the left $Z G$-module $B_{3}(\bar{C} / \widetilde{C})$ of relative 3-boundaries. Hence,

$$
\left(\boldsymbol{U}_{\square}: \sum_{U_{\square} \in U_{\square}}\left(\partial W_{\square} / \partial U_{\square}\right) U_{\square}=0, \forall W_{\square} \in \boldsymbol{W}_{\square}\right)
$$

is a presentation of $H_{3}(\bar{C} / \widetilde{C})$ as a left $Z G$-module. Thus using the isomorphism $\partial_{*}$ taking $U_{\square}$ to $\partial U_{\square}$, we have the first presentation.

Next, let $\widetilde{C}^{(3)}$ be a left $Z G$-chain complex such that $\widetilde{C}^{(3)}$ and $\widetilde{C}$ agree up to and including $\widetilde{C}_{3}$ and such that $\widetilde{C}_{q}^{(3)}=0$ for $q>3$. Then $H_{2} \widetilde{C} \cong H_{2} \widetilde{C}^{(3)}$ and by the same argument, $H_{2} \widetilde{C}^{(3)}=H_{3}\left(\bar{C} / \widetilde{C}^{(3)}\right)$, which gives the second presentation.

Finally, by Definition 6.2 , the obstruction $k(\bar{C}, \widetilde{C})$ is represented by

$$
k=\eta i \partial_{3}: \bar{C}_{3} \longrightarrow Z_{2} \bar{C}=Z_{2} \widetilde{C} \longrightarrow H_{2} \widetilde{C}
$$

Hence,

$$
k\left(U^{*}\right)=\partial U^{*}=\left\{\begin{array}{cl}
\partial U^{*} & \text { if } U^{*} \in \boldsymbol{U}_{\square} \\
0 & \text { if } U^{*} \in \boldsymbol{U}_{-} \cup \boldsymbol{U}_{0} \cup \boldsymbol{U}_{+}
\end{array}\right.
$$

since $\partial U=0$ in $H_{2} \widetilde{C}$ for all $U \in \boldsymbol{U}_{-} \cup \boldsymbol{U}_{0} \cup \boldsymbol{U}_{+}$.

The following theorem will be used to simplify 2-knot and 3-manifold calculations.

${ }^{6}$ In the termonology of $[\mathbf{4 3}], H_{2}(G ; Z G) \cong H_{3}(G, G ; Z G)$. 
THEOREM 9.3. Let $\left(K, K_{-}, K_{+}\right)$be an aspherical splitting of a $\mathrm{CW}$ complex $K$ (see Def. 7.1) and let $G$ be the associated group system (see Def. 3.2). Hence, by Theorem 3.2, $\pi_{1} K=\lim G$. Let $\boldsymbol{C}$ be a Fox-Lyndon resolution system over $\boldsymbol{G}$ corresponding to the presentation $\left(\boldsymbol{x}_{-} ; \boldsymbol{x}_{0} ; \boldsymbol{x}_{+}: \boldsymbol{r}_{-} ; \boldsymbol{r}_{0} ; \boldsymbol{r}_{+}\right)$. If $K_{0}=K_{-} \cap K_{+}$collapses to $a$ 2-dimensional $\mathrm{CW}$ complex and if $\left(\boldsymbol{x}_{0}: \boldsymbol{r}_{0}\right)$ is aspherical, (i.e., its associated 2-complex is aspherical), then $\mathrm{Ker} \partial_{2}^{0}=0$, i.e., $\boldsymbol{U}_{0}$ of Theorem 9.2 is vacuous. Hence, we may also assume $\operatorname{Ker} \partial_{3}^{0}=0$, i.e., $W_{0}$ of Theorem 9.2 is vacuous.

$X$. What is the significance of the algebraic 2-type of 2knots? Throughout this section (unless stated otherwise), $K$ and $K^{\prime}$ will denote the exteriors of $n$-knots. (See notation in $\S 0$.)

We now attempt to understand how significant are the remaining homotopy groups $\pi_{q} K$ for $q \geqq 3$ for 2-knots. A complete answer to this question would indeed be a four dimensional analogue of the asphericity of 1-knots [53]. The evidence given herein (Theorem 10.1) suggests that the algebraic 2 -type ${ }^{7}$ completely determines the homotopy type of 2 -knot complements. If this is so, then the groups $\pi_{q} K$ for $q \geqq 3$ are of little significance.

Since the asphericity of 1-knots essentially means that the algebraic 1-type (i.e., the fundamental group) completely determines the homotopy type of 1-knot complements, we now ask if the analoguous property is true of 2 -knots, i.e.,

QUESTION. If the complements of two smooth (or locally flat PL) 2-knots are of the same algebraic 2-type ${ }^{7}$, then are their complements of the same homotopy type?

In an attempt to gain some insight into the above question, we give the following definition.

DEFINITION 10.1. An $n$-knot is quasi-aspherical (QA) if the $(n+1)$-th homology group of the universal cover of its exterior vanishes.

THEOREM 10.1. Let $K$ and $K^{\prime}$ be the exteriors of two smooth, (or locally flat PL) QA 2-knots. Then $K$ and $K^{\prime}$ are of the same homotopy type if and only if they are of the same algebraic 2-type.

Proof. The exteriors $K$ and $K^{\prime}$ are homotopic to 3-dimensional CW complexes, which we also 'denote by the same symbols $K$ and $K^{\prime}$, respectively. Hence, we need only prove that, if $K$ and $K^{\prime}$

\footnotetext{
${ }^{7}$ In [48], S. MacLane and J.H. C. Whitehead call this the algebraic 3-type.
} 
are two 3-dimensional CW complexes of the same algebraic 2-type, then $K$ and $K^{\prime}$ are of the same homotopy type.

Assume that $K$ and $K^{\prime}$ are of the same algebraic 2-type. Hence, there exists an isomorphism $\left(f_{0}, h\right)$ of $\boldsymbol{T}(K)=\left(\pi_{1} K, \pi_{2} K, \boldsymbol{k} K\right)$ onto $T\left(K^{\prime}\right)=\left(\pi_{1} K^{\prime}, \pi_{2} K^{\prime}, k K^{\prime}\right)$ [48]; and by Theorem 3 of [48] this isomorphism has a geometric realization $\phi: K \rightarrow K^{\prime}$.

Let $K^{i}$ and $K^{\prime i}$ denote the $i$-skeletons of $K$ and $K^{\prime}$, p: $\widetilde{K} \rightarrow K$ and $\boldsymbol{p}^{\prime}: \widetilde{K}^{\prime} \rightarrow K$ the universal covers, and $\widetilde{K}^{i}=\boldsymbol{p}^{-1} K^{i}$ and $\widetilde{K}^{\prime i}=\boldsymbol{p}^{-1} K^{\prime i}$.

Following [48], we define an augmentable chain complex $C=C_{*} K$ by $C_{i} K=H_{i}\left(\widetilde{K}^{i}, \widetilde{K}^{i-1}\right)$ if $i>0$ and by $C_{0} K=H_{0} \widetilde{K}^{0}$ if $i=0$ with boundary operator defined in the obvious way. Let $C^{\sharp}=C_{*}^{\sharp} K$ be the corresponding augmented chain complex. Define $C^{\prime}=C_{*} K^{\prime}$ and $C^{\prime \sharp}=C_{*}^{\sharp} K^{\prime}$ in like manner. Then $C^{\sharp}$ and $C^{\prime \#}$ are acyclic in dimensions less than 2. In fact, because of quasi-asphericity $H_{i} C^{*}=H_{i} \widetilde{K}=$ $0=H_{i} \widetilde{K}=H_{i} C^{\prime \prime}$ if $i \neq 2$, where $\widetilde{K}$ and $\widetilde{K}^{\prime}$ are the universal covers of $K$ and $K^{\prime}$, respectively.

By Theorem 4 of $[48],\left(f_{0}, h\right)$ has a combinatorial realization $\left(f_{0}, \lambda\right): C^{3} \rightarrow C^{\prime 3}$ which induces an isomorshism of $H_{i} C^{\sharp}$ for $i<3$. $\quad\left(C^{3}\right.$ and $C^{\prime 3}$ denote the 3-skeletons of $C$ and $C^{\prime}$ respectively.) But $H_{i} C=$ $0=H_{i} C^{\prime}$ for $i>2$ and $C_{i} K=0=C_{i} K^{\prime}$ for $i>3$. Hence, $\left(f_{0}, h\right)$ has a combinatorial realization $\left(f_{0}, \lambda\right): C \rightarrow C^{\prime}$ which induces an isomorphism of $H_{i} C$ onto $H_{i} C^{\prime}$ for all $i$.

By Theorem 5 of [48], $C \equiv{ }_{i} C^{\prime}$ for all $i$. Thus, $C$ is equivalent to $C^{\prime}$. On the other hand, $K$ and $K^{\prime}$ are $J_{2}$-complexes of dimension 3. So by Theorem 15 of [69], $K$ is of the same homotopy type as $K^{\prime}$.

Actually, we have proven more than stated above, namely:

THEOREM 10.2. If $Q$ and $Q^{\prime}$ are topological spaces such that

(1) $Q$ and $Q^{\prime}$ are dominated by connected 3-dimensional $\mathrm{CW}$ complexes, and

(2) The third homology groups of the universal covers of $Q$ and $Q^{\prime}$ both vanish, then $Q$ and $Q^{\prime}$ are of the same homotopy type if and only if they are of the same algebraic 2-type.

It is easy enough to show that the class of QA knots is immense. It is proven in [26] that:

THEOREM 10.3 [26]. An n-knot is not QA if and only if its fundamental group $G$ has a decomposition of the form $A_{C}^{*} B$ with $C$ finite and properly contained in $A$ and $B$ such that the meridian subgroup $H$ of $G$ lies in $A$. 
TheOREM 10.4. [26,39,41,55,64] The class QA n-knots includes all spun, twist spun, fibered, nilpotent, and finitely ended knots.

If all 2-knots were $\mathrm{QA}$, then the above question would be resolved. However, this is not the case. Recently, Gonzalez-Acuna and Montesinos [26] have constructed an $n$-knot $(n \geqq 2)$ with infinitely many ends, thus solving problem 40 of R.H. Fox [22]. Ratcliffe in [55] has pointed out that this example has a group of the form given in Theorem 10.3 above, and hence, is not QA. In light of Theorem 10.4 above, the examples of [26] are indeed remarkable.

XI. Miscellaneous corollaries on 2-knots. We list below a number of miscellaneous corollaries. The following corollary is an immediate consequence of J. H. C. Whitehead's certain exact sequence [67].

COROLlaRY 11.1. Let $Q$ be a topological space such that

(1) $Q$ is homotopic to a 3-dimensional $\mathrm{CW}$ complex.

(2) The third homology group of the universal cover of $Q$ vanishes.

Then $\pi_{3} Q$ as a $\pi_{1} Q$-module is isomorphic to $\Gamma\left(\pi_{2} Q\right)$, where $\Gamma$ denotes the functor defined in [67]. (See also [45].)

REMARK. If $K$ is the exterior of a smooth (or locally flat PL) QA 2-knot or of a connected QA 3-manifold, then the above corollary can be used to compute $\pi_{3} K$ from $\pi_{2} K$. (For details see [45].)

From $[12,13]$ and from Theorem 10.1 it follows that

COROLLARY 11.2. If $K$ is the exterior of a smooth QA 2-knot and if $\pi_{2} K=0$, then $K$ is a homotopy 1-sphere.

Since all 1-knot groups are of homological and cohomological dimension at most 2 (see $[9,51]$ or Remark 2 in $\S$ VIII), we have:

Corollary 11.3. Let $K$ be the exterior of a spun 2-knot. Then $H^{3}\left(\pi_{1} K_{1} ; \pi_{2} K\right)=0$, and hence, the k-invariant $\boldsymbol{k} K$ vanishes.

Corollary 11.4. Let $Q$ be a connected CW complex whose second homology group vanishes and let $\mathscr{F}$ denote the augmentation ideal of $\pi_{1} Q$. Then the k-invariant $k Q$ lying in $H^{3}\left(\pi_{1} Q \pi_{2} Q\right)$ is nonzero if $\pi_{2} Q / \mathscr{F} \pi_{2} Q \neq 0$.

Proof. By [14], if $k Q=0$, then $\mathscr{F} \pi_{2} Q$ is then the kernel of 
the natural morphism of $\pi_{2} Q$ into $H_{2} Q$.

The following is a consequence of the Leray-Serre spectral sequence. (See $[8$, p. 356].)

Corollary 11.5. Let $Q$ be a CW-complex such that $H_{2} Q=0=$ $H_{3} Q$. Then

$$
H_{3} \pi_{1} Q \cong \pi_{2} Q / \mathscr{F} \pi_{2} Q \quad\left(=Z \bigotimes_{\pi_{1} Q} \pi_{2} Q\right),
$$

where $\mathscr{F}$ denotes the augmentation ideal of $\pi_{1} Q$.

Since every $n$-knot complement is by Alexander duality a homology 1-sphere, we have

COROLLARY 11.6. Let $K$ be the exterior (or complement) of a smooth n-knot. Then

$$
H_{3} \pi_{1} K \cong \pi_{2} K / \mathscr{F} \pi_{2} K \quad\left(=Z \bigotimes_{\pi_{1} K} \pi_{2} K\right),
$$

where $\mathscr{F}$ denotes the fundamental ideal of $K$. Moreover, the $k$ invariant $k K$ lying in $H^{3}\left(\pi_{1} K ; \pi_{2} K\right)$ is nonzero if $H_{3} \pi_{1} K \neq 0$.

The first part of Corollary 11.6 can be thought of as a generalization of the Kervaire condition $H_{2} \pi_{1} Q=0$. (See [36].)

CoROLlaRY 11.7. Let $K$ be the exterior of a smooth (or locally flat PL) fibered 2-knot. Then $k K \neq 0$ if the commutator subgroup $G^{\prime}$ of $\pi_{1} K$ is non-trivial and finite.

Proof. If $G^{\prime}$ is finite, then by Theorem 13 of [3]

$$
\pi_{2} K=\left(\theta:\left(\sum_{g \in G^{\prime}} g\right) \theta=0=(t-1) \theta\right) .
$$

Hence, $\pi_{2} K / \mathscr{F} \pi_{2} K=Z_{n} \neq 0$, where $n$ is the order of $G^{\prime}$, and by Corollary 11.6, $k K \neq 0$.

XII. Conclusion: Problems and more problems. We list below a number of problems which as far as I know are still unresolved.

Problem 1. Let $K$ and $K^{\prime}$ be smooth (or locally flat PL) 2knot exteriors. Is $K$ of the same homotopy type as $K^{\prime}$ iff $K$ and $K^{\prime}$ are of the same algebraic 2-type? (See $\S \mathrm{X}$.) If so, this is a four-dimensional analogue of the asphericity of classical knots. 
Problem 2. Let $K$ and $K^{\prime}$ be smooth $n$-knot exteriors $(n \geqq 3)$. Is $K$ of the same homotopy type as $K^{\prime}$ iff $K$ and $K^{\prime}$ are of the same n-type? ${ }^{8} \quad$ (See $\left.[50,68].\right) \quad$ (See $\left.\S \mathrm{X}.\right)$

Problem 3. (Ratcliffe.) Is a smooth n-knot ( $n \geqq 2)$ quasiaspherical if and only if it is finitely ended? (See Theorem 10.3 and last paragragh of $\S \mathrm{X}$. See also $[39,48,50,55]$.) (See $\S \mathrm{X}$.

Problem 4. (Problem 37 of Fox [22].) Are there any nontrivial smooth (or locally flat PL) aspherical 2-knots? This appears to be a very difficult problem. The reader should note that a smooth (or locally flat PL) 2-knot is aspherical if and only if the fundamental group $\pi_{1} K$ of its exterior $K$ is infinite cyclic. The "only if" part of this statement is proven in $[12,13]$. The "if" part follows from [38, Theorem 3.4]. (See [34, 61].) Moreover, the exterior $K$ of a smooth (or locally flat PL) aspherical 2-knot is a homotopy 1sphere. For a map from $S^{1}$ to $K$ can easily be constructed which induces isomorphisms $\pi_{*} S^{1} \cong \pi_{*} K$. Hence, by a theorem of J.H.C. Whitehead, $K$ is a homotopy $S^{1}$. (See also [25].)

Problem 5. (Problem 8 of Gordon [28].) Let $K$ be the exterior of a smooth (or locally flat PL) 2-knot $\left(S^{4}, k S^{2}\right)$. Is $\pi_{2} K$ always finitely generated as a left $Z \pi_{1} K$-module?

Problem 6. Let $K$ be as in Problem 5. Is $\pi_{2} K$ considered only as a group always a free abelian group? (This is true for all spun 2-knots. See [17].)

Problem 7. Let $K$ be as in Problem 5. Is $k K \neq 0$ if and only if $H_{3} \pi_{1} K \neq 0$. (See Corollaries 11.6 and 11.7.) (From [15], it follows that $H_{3} \pi_{1} K \neq 0$ iff the reduced $K$-invariant is non-zero.)

Problem 8. Let $K$ be as in Problem 5. What is the relationship (if any) between $H_{3} \pi_{1} K$, the number of ends of $\pi_{1} K$, and quasiasphericity? (See $\S 10$ and 11.)

Problem 9. When does a 2-knot diagram represent the trivial 2-knot?

Problem 10. When do two 2-knot diagrams represent the same $2-k n o t ?$

Problem 11. Construct a table of all 2-knot diagrams with

\footnotetext{
8 This is called the $(n+1)$-type in [68].
} 
$\leqq 6$ (or 7 , or $8, \cdots$ ) crossings and with $\leqq 2($ or 3 , or $4, \cdots)$ hyperbolic points. (See Problems 9 and 10 above.)

Problem 12. Is there a CW-complex $K$ such that no cell decomposition of its underlying space $|K|$ has an aspherical splitting? Bill Beckmann [5] has pointed out that every 2-dimensional CW complex has a subdivision which has an aspherical splitting.

Problems 13-16. These problems can be found in the addendum.

Appendix A. A computing manual for algebraic 2-type calculations. If the reader is interested only in computing the algebraic 2 -type of closed connected 3-manifolds and of 2-knot complements, he or she need only read Theorem A2.1 and observations 1 through 3 and the hint, all in part A2 of this appendix. Explicit 2-knot calculations are given in Appendix $B$. If the reader has more general calculations in mind, then all of Appendix A should be read.

Part A1. The computation for arbitrary aspherical triads.

DEFINITION A1.1. Let $\left(K, K_{-} K_{+}\right)$be an aspherical splitting of a CW complex $K$, i.e., a triad of $\mathrm{CW}$-complexes such that

(1) $K=K_{-} \cup K_{+}$.

(2) $K_{-}, K_{0}=K_{-} \cap K_{+}, K_{+}$are pathwise connected.

(3) $K_{-}, K_{0}, K_{+}$are aspherical, i.e., $\pi_{q} K_{\alpha}=0$ for $\alpha=-, 0,+$ and for $q>1$.

Let $\left(\boldsymbol{x}_{0}: \boldsymbol{r}_{0}\right)$ be a presentation of $\pi_{1} K_{0}$, let $\left(\boldsymbol{x}_{0}, \boldsymbol{x}_{ \pm}: \boldsymbol{r}_{0}, \boldsymbol{r}_{ \pm}\right)$be a presentation of $\pi_{1} K_{ \pm}$such that $x_{i 0} \mapsto x_{i 0}$ (for all $x_{i 0} \in \boldsymbol{x}_{0}$ ) is the morphism $g_{ \pm}: \pi_{1} K_{0} \rightarrow \pi_{1} K_{ \pm}$induced by inclusion, and let $g_{\alpha}^{\infty}: \pi_{1} K_{\alpha} \rightarrow \pi_{1} K$ denote the morphisms induced by inclusion for $\alpha=-, 0,+$. It follows from the Van Kampen theorem that $\left(\boldsymbol{x}_{-}, \boldsymbol{x}_{0}, \boldsymbol{x}_{+}: \boldsymbol{r}_{-}, \boldsymbol{r}_{0}, \boldsymbol{r}_{+}\right)$is a presentation of $\pi_{1} K$.

Linearly order $\boldsymbol{x}_{-} \cup \boldsymbol{x}_{0} \cup \boldsymbol{x}_{+}$and $\boldsymbol{r}_{-} \cup \boldsymbol{r}_{0} \cup \boldsymbol{r}_{+}$such that $x_{i-}<x_{j 0}<$ $x_{k+}$ for all $i, j, k$ and $r_{\alpha_{-}}<r_{\beta_{0}}<r_{\gamma+}$ for all $\alpha, \beta, \gamma$ and consider the equations:

$$
\begin{aligned}
& \vec{\lambda}^{0}\left(\frac{\partial \boldsymbol{r}_{0}}{\partial \boldsymbol{x}_{0}}\right)=\overrightarrow{0}, \\
& \vec{\lambda}^{+}\left(\frac{\partial\left(\boldsymbol{r}_{+} \cup \boldsymbol{r}_{0}\right)}{\partial\left(\boldsymbol{x}_{0} \cup \boldsymbol{x}_{+}\right)}\right)=\overrightarrow{0}, \\
& \vec{\lambda}-\left(\frac{\partial\left(\boldsymbol{r}_{0} \cup \boldsymbol{r}_{-}\right)}{\partial\left(\boldsymbol{x}_{-} \cup \boldsymbol{x}_{0}\right)}\right)=\overrightarrow{0},
\end{aligned}
$$$$
(*)
$$ 


$$
\vec{\lambda} \square\left(\frac{\partial\left(\boldsymbol{r}_{+} \cup \boldsymbol{r}_{0} \cup \boldsymbol{r}_{-}\right)}{\partial\left(\boldsymbol{x}_{-} \cup \boldsymbol{x}_{0} \cup \boldsymbol{x}_{+}\right)}\right)=\overrightarrow{0}
$$

where the rows and columns of the above matrices are ordered according to the above constructed linear ordering ${ }^{9}$ and where $\partial r_{i} / \partial x_{j}$ denotes the Fox derivative. (Note: $\left(\partial \boldsymbol{r}_{0} / \partial \boldsymbol{x}_{ \pm}\right),\left(\partial \boldsymbol{r}_{ \pm} / \partial \boldsymbol{x}_{\mp}\right)$ are all zero matrices.)

Let $\boldsymbol{\partial} \boldsymbol{U}_{0}$ denote a set of generators of the left $Z \pi_{1} K_{0}$-module of solutions $\vec{\lambda}^{0}$ of equation $\left({ }^{*} 0\right)$ over the ring $Z \pi_{1} K_{0}$. Each solution of equation $\left({ }^{*} 0\right)$ induces a solution $\vec{\lambda}^{ \pm}$of equation $\left({ }^{*} \pm\right)$ over $Z \pi_{1} K_{ \pm}$. Let $\partial \boldsymbol{U}_{0}$ also denote the induced solutions of $\left({ }^{*} \pm\right)$ over $Z \pi_{1} K_{ \pm}$. Let $\partial U_{ \pm} \cup \partial U_{0}$ denote a set of generators of the left $Z \pi_{1} K_{ \pm}$-module of solutions $\vec{\lambda} \pm$ of equation (* \pm ) over $Z \pi_{1} K_{ \pm}$. Each solution of $(* \pm)$ over $Z \pi_{1} K_{ \pm}$induces a solution of $\left({ }^{*} \square\right)$ over $Z \pi_{1} K$. Let $\boldsymbol{\partial} U_{-} \cup \partial U_{0} \cup$ $\partial U_{+}$also denote these induced solutions of $(* \square)$ over $Z \pi_{1} K$. Finally, let $\boldsymbol{\partial} \boldsymbol{U}_{-} \cup \boldsymbol{\partial} \boldsymbol{U}_{0} \cup \boldsymbol{\partial} \boldsymbol{U}_{\square} \cup \boldsymbol{\partial} \boldsymbol{U}_{+}$denote a set of generators of the left $Z \pi_{1} K$-module of solutions $\vec{\lambda}^{\square}$ of $\left({ }^{*} \square\right)$ over $Z_{1} \pi K$. Then $\partial U_{\square}$ is a set of generators of $\pi_{2} K$ as a left $Z \pi_{1} K$-module.

A complete set of relations among the generators $\partial U_{\square}$ of $\pi_{2} K$ is computed as follows. Linearly order the set $\partial \boldsymbol{U}_{-} \cup \partial \boldsymbol{U}_{0} \cup \partial U_{\square} \cup \partial \boldsymbol{U}_{+}$ such that $\boldsymbol{\partial} \boldsymbol{U}_{\alpha-}<\boldsymbol{\partial} \boldsymbol{U}_{\beta_{0}}<\boldsymbol{\partial} \boldsymbol{U}_{\partial \square}<\boldsymbol{\partial} \boldsymbol{U}_{\gamma+}$ for all $\alpha, \beta, \gamma, \delta$. Let $\boldsymbol{\partial} \boldsymbol{R}_{\alpha}$ be a set of symbols in $1-1$ correspondence with the relators $r_{\alpha}$ for $\alpha=-, 0,+$. Give $\boldsymbol{\partial} \boldsymbol{R}_{-} \cup \boldsymbol{\partial} \boldsymbol{R}_{0} \cup \boldsymbol{\partial} \boldsymbol{R}_{+}$the linear ordering induced by that on $\boldsymbol{r}_{-} \cup \boldsymbol{r}_{0} \cup \boldsymbol{r}_{+}$. Let $\partial U_{o} / \partial R_{l}$ denote the component of the vector $\partial U_{\sigma}$ corresponding to $r_{l}$ for all $\partial U_{\sigma}$ in $\partial U_{-} \cup \partial U_{0} \cup \partial U_{\square} \cup \partial U_{+}$ and for all $r_{l}$ in $r_{-} \cup r_{0} \cup r_{+}$. And consider the equations

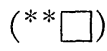

$$
\vec{\mu} \square\left(\frac{\partial U_{+} \cup \partial U_{0} \cup \partial U_{\square} \cup \partial U_{-}}{\partial R_{+} \cup \partial R_{0} \cup \partial R_{-}}\right)=\overrightarrow{0},
$$

where the rows and columns of the above matrices are ordered according to the above constructed linear orderings. ${ }^{10}$ (Note: $\left(\boldsymbol{\partial} \boldsymbol{U}_{0} / \boldsymbol{\partial} \boldsymbol{R}_{ \pm}\right)$, $\left(\boldsymbol{\partial} U_{ \pm} / \boldsymbol{\partial} \boldsymbol{R}_{\mp}\right),\left(\boldsymbol{\partial} U_{ \pm} / \boldsymbol{\partial} \boldsymbol{R}_{\square}\right),\left(\boldsymbol{\partial} U_{0} / \boldsymbol{\partial} \boldsymbol{R}_{\square}\right)$ are all zero matrices. $)$

Let $\partial W$ denote a set of generators of the left $Z \pi_{1} K$-module of solutions $\vec{\mu} \square$ of $\left({ }^{* *} \square\right)$ over $Z \pi_{1} K$. Finally, let $\partial W_{\omega} / \partial U_{\sigma}$ denote the component of $\partial W_{\omega}$ corresponding to $\partial U_{\sigma}$ for all $\partial W_{\omega}$ in $\partial W$. Then

$$
\left\{\sum_{\square \in U_{\square}}\left(\partial W / \partial U_{\square}\right) \partial U_{\square}=0 \mid W \in \boldsymbol{W}\right\}
$$

is a complete set of relations in $\pi_{2} K$ among the generators $\partial U_{\square}$. Hence,

\footnotetext{
${ }^{9}$ Rows in decreasing order from top to bottom. Columns in increasing order from left to right.

${ }^{10}$ Rows ordered as in footnote 9. Columns in decreasing order from left to right.
} 


$$
\left(\left\{\partial U_{\square} \mid \partial U_{\square} \in \partial U_{\square}\right\}:\left\{\sum_{\partial U_{\square} \in \partial U_{\square}}\left(\partial W / \partial U_{\square}\right) \partial U_{\square}=0 \mid \partial W \in \partial W\right\}\right)
$$

is a presentation of $\pi_{2} K$ as a left $Z \pi_{1} K$-module.

Finally, let $W, U, R$ be a set of symbols in $1-1$ correspondence with the elements of $\partial W, \partial U=\partial U_{-} \cup \partial U_{0} \cup \partial U_{\square} \cup \partial U_{+}, \partial R=\partial R_{-} U$ $\boldsymbol{\partial} \boldsymbol{R}_{0} \cup \partial \boldsymbol{R}_{+}$, respectively and let $\bar{C}_{4}, \bar{C}_{3}, \bar{C}_{2}$ be the free left $Z \pi_{1} K$ modules on the sets $W, U, R$, respectively. Then

$$
\bar{C}_{4} \stackrel{\bar{\partial}_{4}}{\longrightarrow} \bar{C}_{3} \stackrel{\bar{\partial}_{3}}{\longrightarrow} \bar{C}_{2}
$$

is a portion of a free resolution over $\pi_{1} K$, where

$$
\begin{aligned}
& \bar{\partial}_{4} W=\sum_{U \in U}(\partial W / \partial U) U, \forall W \in W \quad\left(\text { over } Z \pi_{1} K\right) \\
& \bar{\partial}_{3} U=\sum_{R \in \boldsymbol{R}}(\partial U / \partial R) R, \forall R \in \boldsymbol{R} \quad\left(\text { over } Z \pi_{1} K\right) .
\end{aligned}
$$

A representative of the $k$-invariant $k K$ lying in $H^{3}\left(\pi_{1} K ; \pi_{2} K\right)$ is the equivariant 3-cocycle $k$ given by

$$
k(U)=\left\{\begin{aligned}
\partial U & \text { if } U \in \boldsymbol{U}_{\square} \\
0 & \text { if } U \in U_{-} \cup \boldsymbol{U}_{0} \cup U_{+} .
\end{aligned}\right.
$$

A2. Simplifications that occur for Heegaard and hyperbolic splittings. For the reader who is only interested in computing the algebraic 2-type (i.e., $\pi_{1}, \pi_{2}$, and $k \in H^{3}\left(\pi_{1} ; \pi_{2}\right)$ ) of 2-knot exteriors or of 3-manifolds, we state and prove the following theorem. (The definition of hyperbolic splitting is given in $\S$ II (Def. 2.1). A definition for Heegaard splitting can be found in any standard text on 3-manifolds. See, for example, [32, Chapter 2].)

THEOREM A2.1. Let $\left(K, K_{-}, K_{+}\right)$be a hyperbolic splitting of a 2-knot (or a Heegaard splitting of positive genus of a closed connected 3-manifold). Let $\left(\boldsymbol{x}_{0}: \boldsymbol{r}_{0}\right)$ be an aspherical presentation of $\pi_{1} K_{0}$ (see Observation 1 below) and let $\left(\boldsymbol{x}_{0}: \boldsymbol{r}_{0} \cup \boldsymbol{r}_{ \pm}\right)$be the presentation of $\pi_{1} K_{ \pm}$obtained by adjoining the hyperbolic relators $\boldsymbol{r}_{ \pm}$(see Observation 2 below) [or by adjoining the meridinal handle relators $\boldsymbol{r}_{ \pm}$(see Observation 2 below)]. Let $\boldsymbol{x}=\boldsymbol{x}_{0}$ and $\boldsymbol{r}=\boldsymbol{r}_{-} \cup \boldsymbol{r}_{0} \cup \boldsymbol{r}_{+}$and let $(\partial \boldsymbol{r} / \partial \boldsymbol{x})=\left(\partial r_{\alpha} / \partial x_{\beta}\right)$ denote the Jacobian matrix whose $\alpha \beta$ entry is the image of the Fox derivative $[11,18] \partial r_{\alpha} / \partial x_{\beta}$ in the ring $Z \pi_{1} K$. Let $\partial U_{ \pm}$be a set of generators of the left $Z \pi_{1} K$-module of solutions $\vec{\lambda}$ of equation $(*)$ 
over $Z \pi_{1} K$ such that the components of $\vec{\lambda}$ corresponding to $\boldsymbol{r}_{\mp}$ all vanish. Let $\partial U_{\square}$ be a set of solutions of $\left({ }^{*}\right)$ over $Z \pi_{1} K$ such that $\boldsymbol{\partial} U=\boldsymbol{\partial} U_{-} \cup \partial U_{\square} \cup \partial U_{+}$is a set of generators of the left $Z \pi_{1} K$-module of all solutions $\vec{\lambda}$ of $\left({ }^{*}\right)$.

Next let $\partial R_{\beta}$ be another notation for $r_{\beta}$ and let $\partial U_{\alpha} / \partial R_{\beta}$ denote the component of $\partial U_{\alpha}$ corresponding to $\partial R_{\beta}=r_{\beta}$. Now let $\partial W$ be a set of generators of the left $Z \pi_{1} K$-module of solutions $\vec{\mu}$ of equation (**)

$$
\vec{\mu}(\partial U / \partial R)=0 \text {, }
$$

over $Z \pi_{1} K$.

If $\partial W / \partial U$ denotes the component of $\partial W$ corresponding to $\partial U$ for all $\partial W \in \partial W$ and for all $\partial U \in \partial U$, then

$$
\left(\partial U_{\square}: \sum_{\partial U_{\square} \in \partial U_{\square}}\left(\partial W / \partial U_{\square}\right) \partial U_{\square}=0 \text { for all } \partial W \in \partial W\right)
$$

is a presentation of $\pi_{2} K$ as a left $Z \pi_{1} K$-module.

Finally, let $\boldsymbol{W}, \boldsymbol{U}, \boldsymbol{R}, \boldsymbol{X}$ be sets of symbols in 1-1 correspondence with the sets of symbols $\partial W, \partial \boldsymbol{U}, \boldsymbol{r}, \boldsymbol{x}$, respectively; and let $\bar{C}_{4}, \bar{C}_{3}, \bar{C}_{2}, \bar{C}_{1}$, be respectively the free left $Z \pi_{1} k$-modules on $\boldsymbol{W}, \boldsymbol{U}, \boldsymbol{R}, \boldsymbol{X}$. Let $\bar{C}^{(4)}$ denote the left $Z \pi_{1} K$-chain complex

$$
\bar{C}_{4} \stackrel{\bar{\partial}_{4}}{\longrightarrow} \bar{C}_{3} \stackrel{\bar{\partial}_{3}}{\longrightarrow} \bar{C}_{2} \stackrel{\bar{\partial}_{2}}{\longrightarrow} \bar{C}_{1} \stackrel{\bar{\partial}_{2}}{\longrightarrow} Z \pi_{1} K \stackrel{\varepsilon}{\longrightarrow} Z \longrightarrow 0
$$

given by

$$
\begin{gathered}
\bar{\partial}_{4} W=\sum_{U \in U}(\partial W / \partial U) U, \quad \bar{\partial}_{3} U=\sum_{R \in R}(\partial U / \partial R) R \\
\bar{\partial}_{2} R=\sum_{X \in X}(\partial r / \partial x) X, \bar{\partial}_{1} X=x-1, \quad \varepsilon\left(\sum_{i} n_{i} g_{i}\right)=\sum_{i} n_{i} .
\end{gathered}
$$

Then $\bar{C}^{(4)}$ is a truncation of a free resolution of $\pi_{1} K$. A representative of the $k$-invariant $k K$ lying in $H^{3}\left(\pi_{1} K ; \pi_{2} K\right)$ is the equivariant 3-cocycle $k: \bar{C}_{3} \rightarrow \pi_{2} K$ given by

$$
k(U)=\left\{\begin{array}{l}
\partial U \text { if } U \in \boldsymbol{U}_{\square} \\
0 \text { if } U \in \boldsymbol{U}_{-} \cup \boldsymbol{U}_{+} .
\end{array}\right.
$$

Proof. Theorem 9.2 gives a presentation of $\pi_{2} K$ and a representative of the $k$-invariant. Since the presentation $\left(\boldsymbol{x}_{0}: \boldsymbol{r}_{0}\right)$ of $\pi_{1} K_{0}$ is chosen to be aspherical, it follows from Theorem 9.3 that $\partial U_{0}=$ $\varnothing=\partial W_{0}$.

Moreover, for both Heegaard splittings of closed connected 3-manifolds (see [32]) and hyperbolic splittings of 2-knots (see Theorem 7.4), $\pi_{1} K_{ \pm}$is of homological dimension one. Hence, since 
the morphisms $g_{ \pm}^{\infty}: \pi_{1} K_{ \pm} \rightarrow \pi_{1} K$ induced by inclusion are both epimorphisms, it follows from Theorem 5.4 that the set $\partial U_{ \pm}$can also be obtained by finding a set of generators of the left $Z \pi_{1} K$-module of solutions $\vec{\lambda}$ of $\left(^{*}\right)$ over $Z \pi_{1} K$ (rather than $Z \pi_{1} K_{ \pm}$) such that the components of $\vec{\lambda}$ corresponding to $r_{\mp}$ all vanish.

OBSERVATION 1. There exist aspherical presentations $\left(\boldsymbol{x}_{0}: \boldsymbol{r}_{0}\right)$ of $\pi_{1} K_{0}$. (A presentation is aspherical if its associated 2-complex is aspherical.) Such presentations are easy enough to find. For Heegaard splittings the usual presentation of the fundamental group $\pi_{1} K_{0}$ of the 2-manifold $K_{0}$ can be chosen. (See [7].) For hyperbolic splittings, the Wirtinger presentation of $\pi_{1} K_{0}$ with one relator deleted is aspherical. (See [11].) Moreover, any presentation derived from an aspherical presentation by applying a finite sequence of Tietze (II) $)^{ \pm 1}$ transformations [11] is aspherical. There are many other transformations which preserve presentation asphericity. (See $[41,42]$.

OBSERVATION 2. Since $g_{-}: \pi_{1} K_{0} \rightarrow \pi_{1} K_{-}$and $g_{+}: \pi_{1} K_{0} \rightarrow \pi_{1} K_{+}$are both epimorphisms, a presentation of $\pi_{1} K_{ \pm}$of the form $\left(\boldsymbol{x}_{0}: \boldsymbol{r}_{0}, \boldsymbol{r}_{ \pm}\right)$ can be obtained from a presentation $\left(\boldsymbol{x}_{0}: \boldsymbol{r}_{0}\right)$ by adding relators $\boldsymbol{r}_{ \pm}$. For Heegaard splittings simply add one relator for each handle, i.e., the relator carried by a meridinal 2-cell of each handle. For hyperbolic splittings of 2-knot exteriors, simply adjoin one relator for each hyperbolic point, i.e., adjoin the upper (if + ) or lower (if - ) hyperbolic relators. (See Fig. 8.)

ObSERVATION 3. Since the groups $\pi_{1} K_{ \pm}$are free for both $r_{+}=a b^{-1}$ or $c d^{-1}$ $r_{+}=a d^{-1}$ or $b c^{-1}$
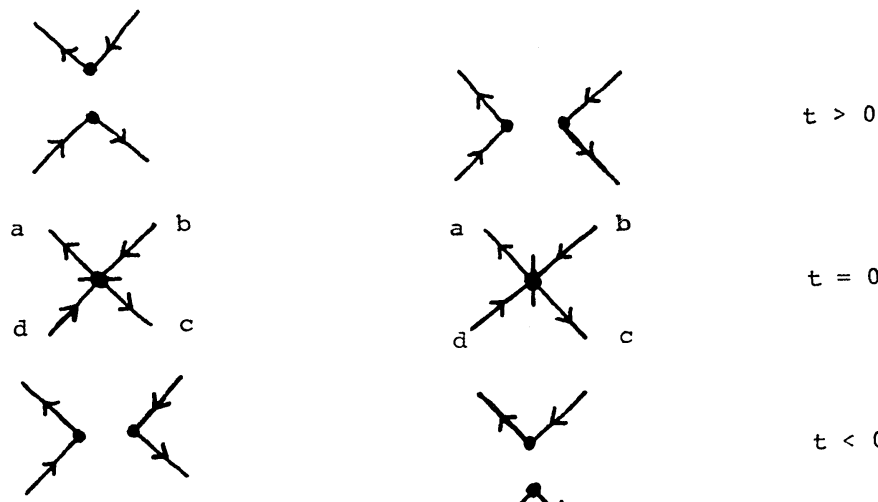

$t<0$

$r_{-}=a d^{-1}$ or $b c^{-1}$

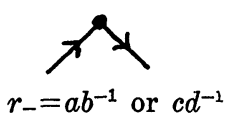

FIGURE 8. Hyperbolic relators. 
Heegaard splittings of 3-manifolds (see [32]) and hyperbolic splittings of 2-knots (see Theorem 7.4), the reader may find it easier to find the $\partial U_{ \pm}$as follows. First, find a set $\partial U_{ \pm}^{\prime}$ of generators of the left $Z \pi_{1} K_{ \pm}$-module of solutions $\vec{\lambda}$ of $\left(^{*}\right)$ over $Z \pi_{1} K_{ \pm}$for which the components of $\vec{\lambda}$ corresponding to $r_{\mp}$ all vanish. Then $\partial U_{ \pm}$is simply the set of solutions of $\left(^{*}\right)$ over $Z \pi_{1} K$ induced by $\partial U_{ \pm}^{\prime}$.

The reader may find the following Hint of use in computing the algebraic 2-type.

HiNT. (Solving equations over group rings.)

Let

$$
\left(\sum_{i} x_{i} a_{i}\right) d=0
$$

be a linear equation in unknowns $\left\{x_{i}\right\}$ over a group ring $Z G$ (i.e., $d \in Z G$ and $\left.a_{i} \in Z G \forall i\right)$.

Case 1. If $d$ is not a divisor of zero, then equation (1) can be replaced by

$$
\sum_{i} x_{i} a_{i}=0 \text {. }
$$

(The element $d$ can be shown not to be a divisor of zero by the methods of $[66, \S 8]$.)

Case 2. If $d$ is a divisor of zero, then equation (1) can be replaced by the equation

$$
\sum_{i} x_{i} a_{i}=\sum_{j} b_{j} d_{j}^{+}
$$

where $\left\{d_{j}^{+}\right\}$is a set of generators of the right ideal of left annihilators of $d$ and where the $b_{j}$ 's are arbitrary.

\section{Appendix B. Explicit calculations for 2-knots.}

We now use Theorem A2.1 of Appendix A to compute the algebraic 2-type of a number of 2-knots. (The methods of [45] can be used to compute $\pi_{3}$ of these examples. See Cor. 11.1 above and following remark. From [49], the Euler characteristic of the embedded connected surface is the number of elliptic points minus the number of hyperbolic points.)

EXAMPLE 1. The diagram of a spun trefoil is given in Figure 5 page 355. (This is Example 9 of [21] and Example 1 of [41, 42].)

A presentation of $\pi_{1} K_{0}$ is $\left(a, b, c, z: r_{0}\right)$, where 


$$
r_{0}=c a c a^{-1} c^{-1} b a b^{-1} a^{-1} b^{-1}
$$

The hyperbolic relators $\boldsymbol{r}_{+}$and $\boldsymbol{r}_{-}$are:

$$
\left\{\begin{array} { l } 
{ r _ { + 1 } = b c ^ { - 1 } } \\
{ r _ { + 2 } = z b a ^ { - 1 } b ^ { - 1 } }
\end{array} \quad \left\{\begin{array}{l}
r_{-1}=z a^{-1} b^{-1} a \\
r_{-2}=b c^{-1} .
\end{array}\right.\right.
$$

Hence, $\left(a, b, c, z ; r_{-2}, r_{-1}, r_{0}, r_{+1}, r_{+2}\right)$ which simplifies to

$$
\left(a, b: a b a b^{-1} a^{-1} b^{-1}\right)
$$

is a presentation of $\pi_{1} K$. Thus equation $\left({ }^{*}\right)$ of Theorem A2.1 becomes

$$
\overrightarrow{0}=\left(\lambda_{2}, \lambda_{1}, \lambda_{0}, \lambda_{-1}, \lambda_{-2}\right)\left(\begin{array}{ccccc}
(\mathrm{a}) & (\mathrm{b}) & (\mathrm{c}) & (\mathrm{z}) \\
-b & b a b^{-1}-1 & 0 & 1 & \left(r_{2}\right) \\
0 & 1 & -1 & 0 & \left(r_{1}\right) \\
0 & -1+a-b a & 1-a+b a & 0 \\
\hdashline a^{-1}-a^{-1} b & -a^{-1} & 0 & 1 \\
0 & 1 & -1 & 0 & \left(r_{0}\right) \\
\left(r_{-1}\right)
\end{array}\right.
$$

By the methods of the Hint in Appendix A, the solutions $\partial U_{-} \cup \partial U_{\square} U$ $\partial U_{+}$of equation $\left(^{*}\right)$ of Theorem A2.1 are found to be:

$$
\begin{cases}\partial U_{+1}=(0,1-a+b a, & 1,0,0) \\ \partial U_{\square}=(0,1, & 0,0,-1) \\ \partial U_{-1}=(0,0, & 1,0,1-a+b a) .\end{cases}
$$

Thus equation $\left({ }^{*}\right)$ of Theorem A2.1 becomes

$$
\overrightarrow{0}=\left(\mu_{+1}, \mu_{\square}, \mu_{-1}\right)\left(\begin{array}{c}
\partial U_{+1} \\
\partial U_{\square} \\
\partial U_{-1}
\end{array}\right) .
$$

By the methods of the Hint in Appendix A, the solutions $\partial \boldsymbol{W}$ of equation $\left(^{* *}\right)$ of Theorem A.1 are:

$$
\partial W=(1,-1+a-b a,-1)
$$

Hence,

$$
\pi_{2} K=\left(\partial U_{\square}:(1-a+b a) \partial U_{\square}=0\right)
$$

and

$$
H^{3}\left(\pi_{1} K ; \pi_{2} K\right)=0 \text {, and hence } k K=0
$$

ExAmple $2(n)$. The diagram of Example $2(n)$ is given in Figure 
6, page 356. (This is Example 12 and 15 of [21] and Example 2 of $[41,42]$.)

A presentation of $\pi_{1} K_{0}$ is

$$
\left(a, x, z_{+}, z_{-}: r_{0}\right)
$$

where

$$
r_{0}=u^{n} z_{-} u^{-n} x^{-1} a^{-1} z_{+}^{-1} x
$$

and

$$
u=z_{-}^{-1} a^{n+1} x a^{-(n+1)} z_{+}^{-1} x .
$$

The hyperbolic relators $\boldsymbol{r}_{+}$and $\boldsymbol{r}_{-}$are:

$$
\left\{\begin{array} { l } 
{ \boldsymbol { r } _ { + 1 } = z _ { + } a ^ { n + 1 } x ^ { - 1 } a ^ { - n } } \\
{ \boldsymbol { r } _ { + 2 } = z _ { - } x ^ { - 1 } }
\end{array} \quad \left\{\begin{array}{l}
\boldsymbol{r}_{-1}=z_{+} x^{-1} \\
r_{-2}=z_{-} a^{n} x^{-1} a^{-(n+1)}
\end{array}\right.\right.
$$

Hence,

$$
\left(a, x: a^{2 n+1}=1, a x=x a^{-1}\right)
$$

is a presentation of $\pi_{1} K$. Thus, by the methods of Hint in Appendix A, the solutions $\partial U_{-} \cup \partial U_{\square} \cup \partial U_{+}$of equation $\left(^{*}\right)$ of Theorem A2.1 are:

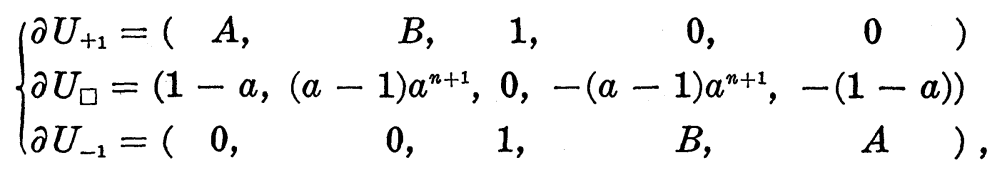

where

$$
\left\{\begin{array}{l}
A=x^{-1} \sum_{0}^{n-1} a^{i}-\sum_{1}^{n+1} a^{i} \\
B=x^{-1} \sum_{0}^{n} a^{i}-\sum_{2}^{n+1} a^{i}
\end{array}\right.
$$

The solutions $\partial W$ of equation (**) of Theorem A2.1 are:

$$
\left\{\begin{array}{l}
\partial W_{1}=\left(1-a, x^{-1} a^{-1} \sum_{0}^{n-1} a^{i}+\sum_{1}^{n+1} a^{i},-(1-a)\right) \\
\partial W_{2}=\left(\begin{array}{lcc}
0, & \sum_{0}^{2 n} a^{i}, & 0
\end{array}\right) .
\end{array}\right.
$$

(See Hint in Appendix A.)

Hence,

$$
\pi_{2} K=\left(\partial U_{\square}:\left(x^{-1} a^{-1} \sum_{0}^{n-1} a^{i}+\sum_{1}^{n+1} a^{i}\right) \partial U_{\square}=0=\left(\sum_{0}^{2 n} a^{i}\right) \partial U_{\square}\right)
$$


which simplifies to

$$
\pi_{2} K=\left(\partial U_{\square}:(x-1) \partial U_{\square}=0=\left(\sum_{0}^{2 n} a^{i}\right) \partial U_{\square}\right),
$$

and

$$
k K=t,
$$

where

$$
H^{3}\left(\pi_{1} K ; Z \pi_{2} K\right)=Z_{2 n+1}=\left(t: t^{2 n+1}=1\right) .
$$

Example 3. The diagram of Example 3 is given in Figure 7, page 9. (This is Example 10 of [21] and Example 3 of $[41,42]$.)

$$
\begin{aligned}
& \pi_{1} K_{0}=\left(a, x, y, z: r_{0}\right) \\
& r_{0}=a y z^{-1} x z y^{-1} x^{-1} a^{-1} y z^{-1} \\
& \left\{\begin{array} { l } 
{ r _ { + 1 } = a x a ^ { - 2 } y ^ { - 1 } } \\
{ r _ { + 2 } = y z ^ { - 1 } }
\end{array} \quad \left\{\begin{array}{l}
r_{-1}=y z^{-1} \\
r_{-2}=x z^{-1}
\end{array} .\right.\right. \\
& \pi_{1} K=\left(a, x: a x=x a^{2}\right) .
\end{aligned}
$$

By the methods found in the Hint of Appendix A, we have:

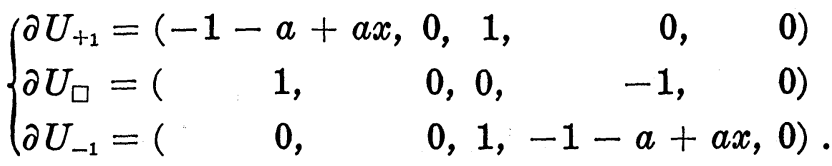

$$
\begin{aligned}
& \partial W=(1,1+a-a x,-1) \text {. }
\end{aligned}
$$

Hence,

$$
\pi_{2} K=\left(\partial U_{\square}:(1+a-a x) \partial U_{\square}=0\right),
$$

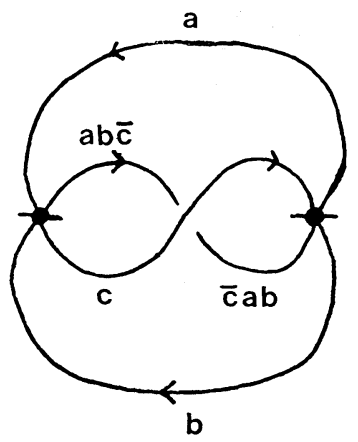

FIGURE 9. Diagram of an embedding of $R P^{2}$ in $S^{4}$.

$\pi_{1}\left(S^{4}-R P^{2}\right)=\left(a: a^{2}\right)=Z_{2}$

$\pi_{2}\left(S^{4}-R P^{2}\right)=\left(\partial U_{\square}:(1+a) \partial U_{\square}=0\right)=Z$ (twisted action) 
and

$$
H^{3}\left(\pi_{1} K ; \pi_{2} K\right)=0 \text { and } k K=0 .
$$

EXAMPLE 4. The diagram of an embedding in $S^{4}$ of the real projective plane $R P^{2}$ is given in Figure 9. Using the methods of Appendix A, we have

$$
\left\{\begin{array}{l}
\pi_{1}\left(S^{4}-R P^{2}\right)=\left(a, b, c: a b^{-1}, a c^{-1}, a b\right)=\left(a: a^{2}\right)=Z_{2} \\
\left.\pi_{2}\left(S^{4}-R P^{2}\right)=\left(\partial U_{\square}:(1+a) \partial U_{\square}=0\right)=Z \text { twisted action }\right) .
\end{array}\right.
$$

(See [54].)

\section{Addendum (Section XII continued)}

Problem 13. Let $K$ be the exterior of a smooth (or locally flat PL) 2-knot $\left(S^{4}, K S^{2}\right)$. Is $\pi_{2} K$ always finitely related as a left $Z \pi_{1} K$ module?

Problem 14. Let $K$ be as in Problem 13. If $\pi_{2} K$ vanishes, then is $K$ always a spherical? (See Problem 4.)

Problem 15. Does there exist a 2-knot whose fundamental group has no non-trivial elements of finite order but whose K-invariant is non-zero? (See Corollary 11.7.)

Problem 16. Do there exist two 2-knots with exteriors $K$ and $K^{\prime}$ respectively such that $\pi_{1} K \cong \pi_{1} K^{\prime}$ and $\pi_{2} K \cong \pi_{2} K^{\prime}$ (as left $Z \pi_{1} K$ modules) but with distinct K-invariants? (See [27].)

\section{REFERENCES}

1. J. J. Andrews and M. L. Curtis, Knotted 2-spheres in the 4-sphere, Ann. of Math., 70 (1959), 565-571.

2. J. J. Andrews and S. L. Lomonaco, The second homotopy group of spun 2-spheres in 4-space, Ann. Math., 90, No. 2 (1969), 199-204.

3. J. J. Andrews and D. W. Sumners, On Higher-dimensional fibered knots, A.M.S. Trans., 153 (1971), 415-426.

4. E. Artin, Zur Isotopie Zweidimensionaler Flächen in $R^{4}$, Abh. Math. Seminar Univ. Hamburg, 4 (1925), 174-177.

5. William Beckmann, Personal communication.

6. Joan S. Birman, Braids, links, and mapping class groups, Annals of Math. Studies, No. 82, Princeton University Press, Princeton, N. J., 1974.

7. S. S. Cairns, Introductory Topology, Ronald, New York, N. Y., 1962.

8. Henri Cartan and Samuel Eilenberg, Homological Algebra, Princeton University Press, Princeton, N. Y., 1956.

9. Richard H. Crowell, $H_{2}$ of subgroups of knot groups, Illionis J. Math., (1970), 665-673. 10. Richard H. Crowell, On the Van Kampen theorem, Pacific J. Math., 9, No. 1 (1959), 43-50. 
11. Richard H. Crowell and Ralph H. Fox, Introduction to Knot Theory, SpringerVerlag, New York, 1977.

12. Eldon Dyer and A. T. Vasquez, The asphericity of higher dimensional knots, Canad. J. Math., 25, No. 6 (1973), 1132-1136.

13. Beno Eckmann, Aspherical manifolds and higher-dimensional knots, Comment. Math. Helvetici, 51 (1976), 93-98.

14. Samuel Eilenberg and Saunders MacLane, Determination of the second homology and cohomology groups of a space by means of homotopy invariants, Proc. Nat. Acad. Sci., (1948), 277-280.

15. - Homology of spaces with operators II, Amer. Math. Soc. Trans., 65, No. 1 (1949), 49-99.

16. Samuel Eilenberg and Norman Steenrod, Foundations of Algebraic Topology, Princeton University Press, Princeton, N. J., 1952.

17. D. B. A. Epstein, Linking spheres, Proc. Camb. Phil. Soc., 56, Part 3 (1960), 215-219.

18. Ralph H. Fox, Free differential calculus. I Derivation in the free group ring, Ann. of Math., 57, No. 3 (1953), 547-560.

19. - Free differential calculus. II The isomorphism problem of groups, Ann. of Math., 59 No. 2 (1954), 196-210.

20. - Free differential calculus. $V$ The Alexander matrices re-examined, Ann. of Math., 71, No. 2, (1960), 408-422.

21. Ralph H. Fox, A quick trip through knot theory, in "Topology of 3-Manifolds and Related Topics," edited by M. K. Fort, Jr., Prentice-Hall, Inc., Englewood Cliffs, N.J., (1962), 120-167.

22. Some problems in knot theory, in "Topology of 3-Manifolds and Related Topics," edited by M. K. Fort, Jr., Prentice-Hall, Inc., Englewood Cliffs, N.J., (1962), 168-176.

23. R. H. Fox and J. W. Milnor, Singularities of 2-spheres in 4-space and equivalence of knots, (unpublished version).

24. — Singularities of 2-spheres in 4-space and cobordism of knots, Osaka J. Math., 3 (1966), 257-267.

25. H. Gluck, The embeddings of two-spheres in the four-sphere, Amer. Math. Soc. Trans., 104 (1962), 308-333.

26. F. González-Acuna and J. M. Montesinos, Ends of knots, Ann. of Math., 108, No. 1 (1978), 91-96.

27. C. McAGordon, Some higher-dimensional knots with the same homotopy groups, Quart. J. Math. Oxford, 24, No. 95 (1973), 411-422.

28. - Problems, "Knot Theory," edited by J. C. Hausmann, Springer-Verlag, New York, (1978), 309-311.

29. M. A. Gutierrez, An exact sequence calculation for the second homotopy of a knot, Amer. Math. Soc. Proc., 32, No. 2 (1972), 571-577.

30. M. A. Gutierrez, An exact sequence calculation for the second homotopy group. II, Amer. Math. Soc. Proc., 40, No. 1 (1973), 327-330.

31. John R. Harper and S. J. Lomonaco, Jr., The k-invariant, in preparation.

32. John Hempel, 3-Manifolds, Annals of Math. Studies, No. 86, Princeton Univ. Press, Princeton, N. J., 1976.

33. Sze-Tsen Hu, Homotopy Theory, Academic Press, New York, 1959.

34. A. Kawauchi, On partial Poincare duality and higher-dimensional knots with $\pi_{1}=Z$, preprint, Kobe University.

35. M. Kervaire, Les noeds de dimensions supérieures, Bull. Soc. Math. France, 93 (1965), 225-271.

36. - On higher dimensional knots, "Differential and Combinororial Topology," Edited by Steward S. Cairns, Princeton Univ. Press, Princeton, N. J., (1965), 105-119. 37. M. Kervaire and Cl. Weber, A survey of multidimensional knots, "Knot Theory," 
edited by J. C. Hausmann, Springer-Verlag, New York, (1978), 61-134.

38. Jerome Levine, Knot modules I, A.M.S. Trans., 229 (1977), 1-50.

39. S. J. Lomonaco, Jr., Finitely ended knots are quasi-aspherical, submitted.

40. - The fundamental ideal and $\pi_{2}$ of higher dimensional knots, Amer. Math.

Soc. Proc., 38, No. 2, (1973), 431-433.

41. The homotopy groups of knots. I. The four dimensional analogue of the asphericity of knots, (manuscript), version 9-14-77.

42. - The homotopy group of knots. II. A solution to problem 36 of R.H. Fox, (manuscript), version 9-14-77.

43. - The homology of group systems with applications to low dimensional topology, A.M.S. Bull., 3, No. 3 (1980), 1049-1052.

44. - The second homotopy group of a spun knot, Topology, 8 (1969), 95-98.

45. - The third homotopy group of some higher dimensional knots, in "Knots, Groups and 3-Manifolds" edited by L.P. Neuwirth, Annals of Math. Studies, Princeton Univ. Press, Princeton, N. J., (1975), 35-43.

46. Roger C. Lyndon, Cohomology theory of groups with a single defining relation, Ann. Math., 52, No. 3 (1950), 650-665.

47. Saunders MacLane, Homology, Academic Press, New York, N. Y., 1963.

48. Saunders MacLane and J. H. C. Whitehead, On the 3-type of a complex, Proc. Nat. Acad. Sci., 36 (1950), 41-48.

49. J. Milnor, Morse Theory, Annals of Math. Studies, No. 51, Princeton Univ. Press, Princeton, N. J., 1963.

50. Robert E. Mosher and Martin C. Tangora, Cohomology Operations and Applications in Homotopy Theory, Harper \& Row, N. Y., 1968.

51. L. P. Neuwirth, Knot Groups, Annals of Math. Studies, No. 56, Princeton Univ. Press, Princeton, N. J., 1965.

52. D. G. Northcott, An Introduction to Homological Algebra, Cambridge Univ. Press, New York, N. Y., 1960.

53. C. D. Papakyriakopoulos, On Dehn's lemma and the asphericity of knots, Ann. Math., 66, No. 1 (1957), 1-26.

54. T. M. Price and D. M. Roseman, Embeddings of the projective plane in four space, manuscript.

55. John Ratcliffe, On the ends of higher dimensional knot groups, Quart. J. Oxford (to appear).

56. Kurt Reidemeister, Complexes and homotopy chains, Amer. Math. Soc. Bull., (1950), 297-307.

57. - Homotopiegruppen von Komplexes, Hamburg. Abh., 10 (1934), 211-215.

58. — Uberdeckungen von Komplexen, J. reine angew. Math., 173 (1935),

164-173.

59. Dale Rolfsen, Knots and Links, Publish or Perish, Berkeley, CA., 1976.

60. Edwin H. Spanier, Algebraic Topology, McGraw-Hill, New York, N. Y., 1966.

61. D. W. Sumners, On asphericity of knots $S^{2}$ in $S^{4}$, preprint.

62. Shinichi Suzuki, Knotting problems of 2-spheres in 4-sphere, Mathematics Seminar Notes, Kobe Univ., 4, No. 3, Nada, Kobe, Japan, (1976).

63. Richard G. Swan, Groups of cohomological dimension one, J. Algebra, 12, (1969), 585-601.

64. G. A. Swarup, An unknotting criterion, J. Pure and Appl. Algebra, 6 (1975). 291-296.

65. H. F. Trotter, Homology of group systems with applications to knot theory, Ann. Math., 76, No. 3, (1962), 464-498.

66. J. H. C. Whitehead, On the asphericity of regions in a 3-sphere, Fund. Math., 32 (1939), 149-166.

67. — A certain exact sequence, Ann. Math., 52 (1950), 51-110.

68. — Combinatorial homotopy I, Amer. Math. Soc. Bull., 55 (1949), 213-245. 
69. J. H. C. Whitehead, Combinatorial homotopy II, Amer. Math. Soc. Bull., 55 (1949), 453-496.

70. The second boundary operator, Proc. Nat. Acad. Sci., 36 (1950), 55-60.

71. E. C. Zeeman, Twisting spun knots, Amer. Math. Soc. Trans., 115 (1965), 471-495.

Received May 21, 1979 and in revised form June 10, 1980. Supported in part by State University of New York Summer Research Grant and also by the L-O-O-P Fund.

State University OF New York

ALbanY, NY 12222

AND

INSTitute FOR Defense ANALyses

Princeton, NJ 08540

Current Address: 7004 South Ridge Drive

Dallas, TX 75214 


\section{PACIFIC JOURNAL OF MATHEMATICS}

\section{EDITORS}

DONALD BABBITT (Managing Editor)

University of California

Los Angeles, CA 90024

HUGo RossI

University of Utah

Salt Lake City, UT 84112

C. C. MOORE and ANDREW OGG

University of California

Berkeley, CA 94720
J. DugundjI

Department of Mathematics

University of Southern California

Los Angeles, CA 90007

R. FinN and J. Milgram

Stanford University

Stanford, CA 94305

\section{ASSOCIATE EDITORS}
R. ARENS
E. F. BECKENBACH
B. H. NeumanN
F. WOLF
K. YOSHIDA

\section{SUPPORTING INSTITUTIONS}

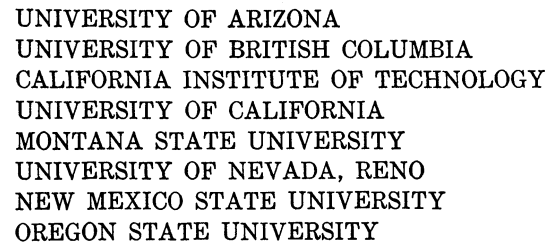

UNIVERSITY OF ARIZONA

UNIVERSITY OF BRITISH COLUMBIA

CALIFORNIA INSTITUTE OF TECHNOLOGY

UNIVERSITY OF CALIFORNIA

MONTANA STATE UNIVERSITY

UNIVERSITY OF NEVADA, RENO

NEW MEXICO STATE UNIVERSITY

OREGON STATE UNIVERSITY

\author{
UNIVERSITY OF OREGON \\ UNIVERSITY OF SOUTHERN CALIFORNIA \\ STANFORD UNIVERSITY \\ UNIVERSITY OF HAWAII \\ UNIVERSITY OF TOKYO \\ UNIVERSITY OF UTAH \\ WASHINGTON STATE UNIVERSITY \\ UNIVERSITY OF WASHINGTON
}

The Supporting Institutions listed above contribute to the cost of publication of this Journal, but they are not owners or publishers and have no responsibility for its content or policies.

Mathematical papers intended for publication in the Pacific Journal of Mathematics should be in typed form or offset-reproduced, (not dittoed), double spaced with large margins. Please do not use built up fractions in the text of the manuscript. However, you may use them in the displayed equations. Underline Greek letters in red, German in green, and script in blue. The first paragraph or two must be capable of being used separately as a synopsis of the entire paper. Please propose a heading for the odd numbered pages of less than 35 characters. Manuscripts, in triplicate, may be sent to any one of the editors. Please classify according to the scheme of Math. Reviews, Index to Vol. 39. Supply name and address of author to whom proofs should be sent. All other communications should be addressed to the managing editor, or Elaine Barth, University of California, Los Angeles, California, 90024.

50 reprints to each author are provided free for each article, only if page charges have been substantially paid. Additional copies may be obtained at cost in multiples of 50 .

The Pacific Journal of Mathematics is issued monthly as of January 1966. Regular subscription rate: $\$ 102.00$ a year (6 Vols., 12 issues). Special rate: $\$ 51.00$ a year to individual members of supporting institutions.

Subscriptions, orders for numbers issued in the last three calendar years, and changes of address shoud be sent to Pacific Journal of Mathematics, P.O. Box 969, Carmel Valley, CA 93924, U.S.A. Old back numbers obtainable from Kraus Per!odicals Co., Route 100, Millwood, NY 10546.

PUBLISHED BY PACIFIC JOURNAL OF MATHEMATICS, A NON-PROFIT CORPORATION

Printed at Kokusai Bunken Insatsusha (International Academic Printing Co., Ltd.). 8-8, 3-chome, Takadanobaba, Shinjuku-ku, Tokyo 160, Japan. 


\section{Pacific Journal of Mathematics}

\section{Vol. 95, No. $2 \quad$ October, 1981}

George E. Andrews, The Rogers-Ramanujan reciprocal and Minc's

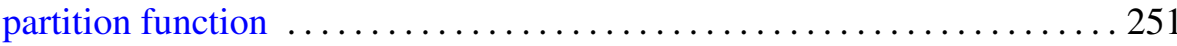

Allan Calder, William H. Julian, Ray Mines, III and Fred Richman,

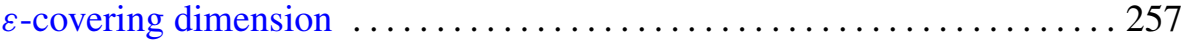

Thomas Curtis Craven and George Leslie Csordas, An inequality for the distribution of zeros of polynomials and entire functions $\ldots \ldots \ldots \ldots 263$

Thomas Jones Enright and R. Parthasarathy, The transfer of invariant

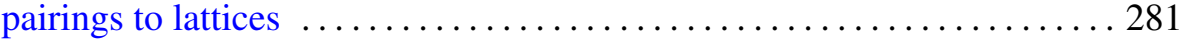

Allen Roy Freedman and John Joseph Sember, Densities and

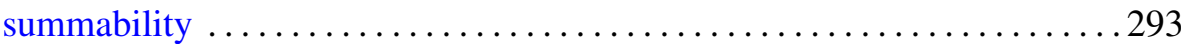

Robert Heller and Francis Aubra Roach, A generalization of a classical necessary condition for convergence of continued fractions . . . . . . 307

Peter Wilcox Jones, Ratios of interpolating Blaschke products ........... 311

V. J. Joseph, Smooth actions of the circle group on exotic spheres ........ 323

Mohd Saeed Khan, Common fixed point theorems for multivalued

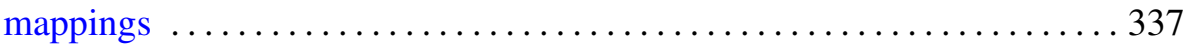

Samuel James Lomonaco, Jr., The homotopy groups of knots. I. How to

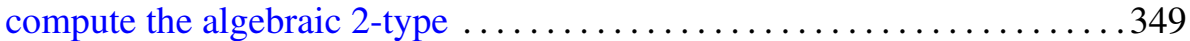

Louis Magnin, Some remarks about $C^{\infty}$ vectors in representations of connected locally compact groups ............................ 391

Mark Mandelker, Located sets on the line . . . . . . . . . . . . . . . . . 401

Murray Angus Marshall and Joseph Lewis Yucas, Linked quaternionic mappings and their associated Witt rings $\ldots \ldots \ldots \ldots \ldots \ldots \ldots \ldots . \ldots \ldots 11$

William Lindall Paschke, $K$-theory for commutants in the Calkin algebra

W. J. Phillips, On the relation $P Q-Q P=-i I$ 435

Ellen Elizabeth Reed, A class of Wallman-type extension. 443

Sungwoo Suh, The space of real parts of algebras of Fourier transforms 461 Antonius Johannes Van Haagen, Finite signed measures on function

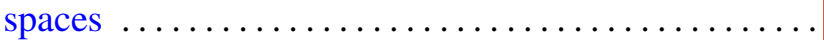

Richard Hawks Warren, Identification spaces and unique uniformity 